2015-08

Flap gate farm: From Venice lagoon

defense to resonating wave energy

production. Part 2: Synchronous

response to incident waves in open sea

Michele, Simone

http://hdl.handle.net/10026.1/17508

10.1016/j.apor.2015.05.002

Applied Ocean Research

Elsevier BV

All content in PEARL is protected by copyright law. Author manuscripts are made available in accordance with publisher policies. Please cite only the published version using the details provided on the item record or document. In the absence of an open licence (e.g. Creative Commons), permissions for further reuse of content should be sought from the publisher or author. 


\title{
Flap gate farm: From Venice lagoon defense to resonating wave energy production. Part 2: Synchronous response to incident waves in open sea
}

\author{
S. Michele ${ }^{\mathrm{a}, *}$, P. Sammarco ${ }^{\mathrm{a}}$, M. d'Errico ${ }^{\mathrm{a}}$, E. Renzi ${ }^{\mathrm{b}}$, A. Abdolali ${ }^{\mathrm{c}}$, G. Bellotti ${ }^{\mathrm{c}}$, F. Dias ${ }^{\mathrm{b}, \mathrm{d}}$ \\ a Department of Civil Engineering and Computer Science, Università degli Studi di Roma “Tor Vergata”, Via del Politecnico 1, 00133 Roma, Italy \\ ${ }^{\mathrm{b}}$ UCD School of Mathematical Sciences, University College Dublin, Belfield, Dublin 4, Ireland \\ ${ }^{\mathrm{c}}$ Department of Civil Engineering, Università di Roma Tre, Via Vito Volterra 62, 00146 Roma, Italy \\ d Centre de Mathématiques et de Leurs Applications (CMLA), Ecole Normale Supérieure de Cachan, 94235, France
}

\section{A R T I C L E I N F O}

\section{Article history:}

Received 25 November 2014

Received in revised form 4 May 2015

Accepted 5 May 2015

Available online 29 May 2015

\section{Keywords:}

Flap gate energy

Wave-body interaction

Resonance

\begin{abstract}
A B S T R A C T
We consider a flap gate farm, i.e. a series of $P$ arrays, each made of $Q$ neighbouring flap gates, in an open sea of constant depth, forced by monochromatic incident waves. The effect of the gate thickness on the dynamics of the system is taken into account. By means of Green's theorem a system of hypersingular integral equations for the velocity potential in the fluid domain is solved in terms of Legendre polynomials. We show that synchronous excitation of the natural frequencies of Sammarco et al. (2013) yields large amplitude response of gate motion. This aspect is fundamental for the optimisation of the gate farm for energy production.
\end{abstract}

(C) 2015 Elsevier Ltd. All rights reserved.

\section{Introduction}

The flap gate systems, i.e. one or more floating bodies hinged at the bottom of the sea and rolling under incoming waves, have recently proved very effective to extract energy from the sea (Whittaker et al. [1]). The mechanical behaviour of a rolling flap gate was initially investigated during the design phase of the storm barriers for protecting Venice Lagoon from flooding. For one array of gates spanning the entire width of a channel, experiments showed that the gates can be excited to oscillate at half the incident wave frequency with a very large amplitude (Mei et al. [2]). In that case, resonance occurs through a nonlinear mechanism when the frequency of the incoming wave is twice the eigenfrequency of the system (Sammarco et al. [3,4]). Li and Mei [5] found the ( $Q-1)$ eigenfrequencies of one array made by $Q$ identical gates spanning the full width of a channel. Later, Sammarco et al. [6] in Part 1 of this paper considered a $P \times Q$ gate farm, and showed that there exist $P \times(Q-1)$ eigenfrequencies and associated modal forms. If the gates are not completely confined in a channel, radiation damping is always present, i.e. wave trapping is imperfect and therefore linear resonance of the eigenmodes is possible (Adamo and Mei [7]).

In this paper a linear theory is developed in order to analyse the resonant behaviour of the $P \times Q$ gate farm in an open sea of constant depth. Unlike in previous models available in the literature (Renzi et al. [8], Renzi and Dias [9-13], Renzi et al. [14,15], Sarkar et al. [16]), all based on the "thin-gate hypothesis" (Linton and Mclver [17]), in this work the gate thickness is assumed finite, i.e. comparable with the other gate dimensions. By means of Green's theorem a system of hypersingular integral equations for the radiation and scattering potential on the boundaries of the gate farm is obtained. Achenbach and Li [18] and Martin and Rizzo [19] adopted a similar procedure to solve crack and acoustic problems, while Parsons and Martin [20-22] used this method to solve scattering and trapping of water waves by rigid plates. Subsequently, Martin and Farina [23] and Farina and Martin [24] used the hypersingular integral equation approach to solve the radiation and scattering problem for a submerged horizontal circular plate.

Here we find the solution in terms of Legendre polynomials. The Haskind-Hanaoka relation is utilised to check the accuracy and the computational cost of the semi-analytical method. We show that in the open sea there are $P \times(Q-1)$ out-of-phase natural modes similar

\footnotetext{
* Corresponding author. Tel.: +390672597016.

E-mail addresses: michele@ing.uniroma2.it (S. Michele), sammarco@ing.uniroma2.it (P. Sammarco), derrico@ing.uniroma2.it (M. d'Errico), emiliano.renzi@ucd.ie (E. Renzi), ali.abdolali@uniroma3.it (A. Abdolali), giorgio.bellotti@uniroma3.it (G. Bellotti), frederic.dias@ucd.ie (F. Dias).
} 


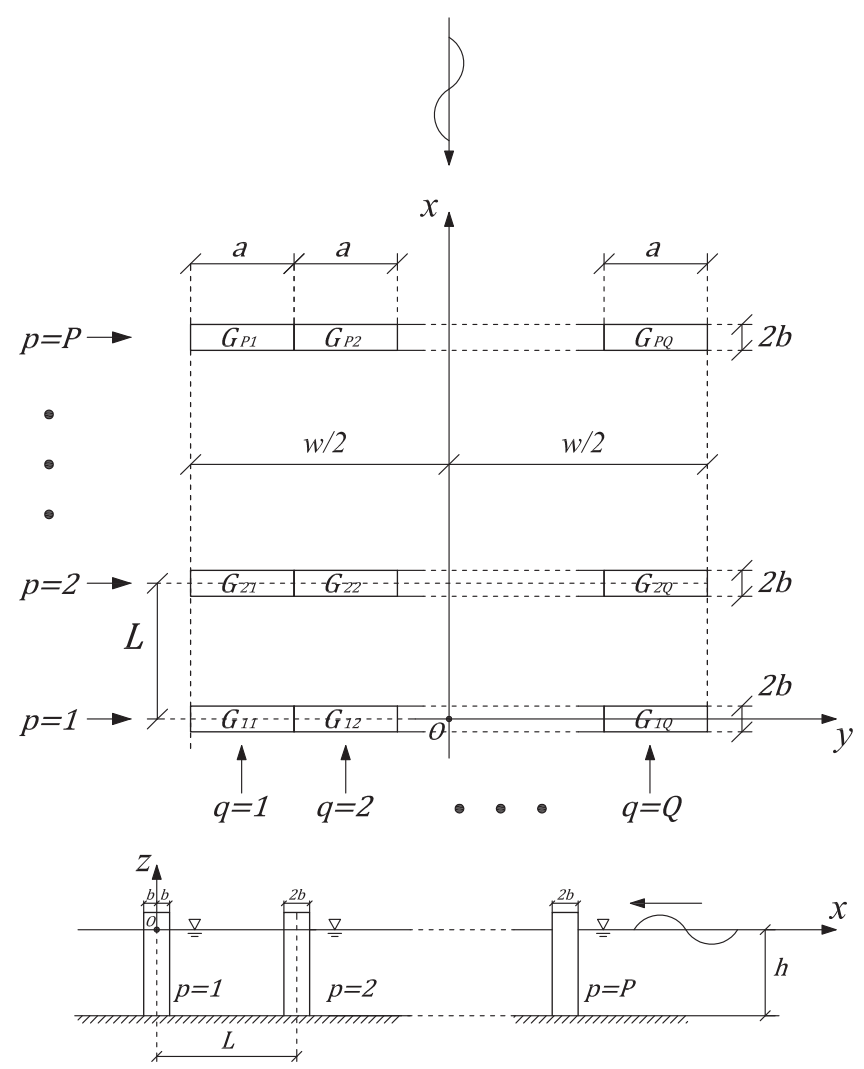

Fig. 1. Plan geometry and side view.

in shape to the case of the gate farm in a channel. The irregular frequencies (Linton and Mclver [17]-Mei et al. [25]) are then evaluated. We also investigate the response of the gate farm to plane incident waves of varying frequency. The gate farm is designed to work in the nearshore, hence normal incidence of the waves is assumed. Large amplitude motions of the gates occur when the incident wave frequency approaches the eigenfrequencies. Hence a linear resonant mechanism of the natural modes in the open sea is effective. Finally, the $P \times Q$ gate farm and a system of $P \times Q$ isolated and independent gates are compared in terms of energy production.

\section{Governing equations for the $P \times Q$ gate farm}

As shown in Fig. 1, consider $P$ arrays of neighbouring flap gates. Each array, $p=1,2, \ldots, P$, is composed by $Q$ identical floating gates $(q=1$, $2, \ldots, Q)$. Let $a$ and $2 b$ be, respectively, the width and the thickness of each gate and let $w=Q a$. Consider a three dimensional Cartesian coordinate system with the $x$ and $y$ axes lying on the mean free surface and the $z$ axis pointing vertically upward. The $y$-axis bisects the first array $(p=1)$, while the $x$-axis is orthogonal to the arrays and is centred among them. All the gates of the pth array are hinged on a common axis lying on $x=(p-1) L, z=-h$, where $L$ is the distance between the arrays and $h$ the sea constant depth. The symbol $G_{p q}$ denotes the $q$ th gate of the $p$ th array, while $\Theta_{p q}$ indicates the angular displacement of $G_{p q}$, positive if clockwise. Monochromatic plane normal incidence waves of amplitude $A$, period $T$ and angular frequency $\omega=2 \pi / T$, coming from $x=+\infty$, force the gates to oscillate back and forth.

Let $\Theta_{p}(y, t)$ indicate the angular displacement function of the $p$ th array:

$$
\Theta_{p}(y, t)=\left\{\Theta_{p 1}(t), \ldots, \Theta_{p q}(t), \ldots, \Theta_{p Q}(t)\right\} .
$$

$\Theta_{p}(y, t)$ is a piece-wise function of $y$, still unknown. The analysis is performed in the framework of irrotational flow and in the limit of small-amplitude oscillations. Therefore, the velocity potential $\Phi(x, y, z, t)$ must satisfy the Laplace equation in the fluid domain $\Omega$ :

$$
\nabla^{2} \Phi=0, \quad(x, y, z) \in \Omega .
$$

On the free surface, the kinematic-dynamic boundary condition reads:

$$
\frac{\partial^{2} \Phi}{\partial t^{2}}+g \frac{\partial \Phi}{\partial z}=0, \quad z=0,
$$

while the no-flux condition on the seabed requires:

$$
\frac{\partial \Phi}{\partial z}=0, \quad z=-h
$$

On the $p=1, \ldots, P$ arrays the kinematic boundary conditions are:

$$
\frac{\partial \Phi}{\partial x}=\frac{\partial \Theta_{p}}{\partial t}(z+h), \quad x=(p-1) L \pm b, y \in\left[-\frac{w}{2}, \frac{w}{2}\right], z \in[-h, 0],
$$




$$
\frac{\partial \Phi}{\partial y}=0, \quad x \in[(p-1) L-b,(p-1) L+b], y= \pm \frac{w}{2}, z \in[-h, 0] .
$$

Note that the no flux condition (6) is given on the finite edges of each array facing the open sea, without channel walls. The time dependence of $\Phi$ and $\Theta_{p}$ can be separated by assuming a harmonic motion of given frequency $\omega$ :

$$
\begin{aligned}
& \Phi(x, y, z, t)=\operatorname{Re}\left\{\phi(x, y, z) e^{-\mathrm{i} \omega t}\right\}, \\
& \Theta_{p}(y, t)=\operatorname{Re}\left\{\theta_{p}(y) e^{-\mathrm{i} \omega t}\right\} .
\end{aligned}
$$

\section{Semi-analytical solution}

The linearity of the problem allows the following decomposition of the potential $\phi(x, y, z)$ :

$$
\phi=\phi^{I}+\phi^{S}+\sum_{p=1}^{P} \sum_{q=1}^{Q} \phi_{p q}^{R}
$$

where

$$
\phi^{I}=-\frac{\mathrm{i} A g}{\omega} \frac{\operatorname{ch} k(h+z)}{\operatorname{ch} k h} e^{-\mathrm{i} k x},
$$

is the potential of the plane incident waves incoming from $x=+\infty, \phi^{S}$ is the potential of the scattered waves and $\phi_{p q}^{R}$ is the potential of the radiated waves due to the moving gate $G_{p q}$ while all the other gates are at rest. In (10), $k$ denotes the wave number, root of the dispersion relation $\omega^{2}=g k$ th $k h$, while i is the imaginary unit. ch, sh and th indicate shorthand notation respectively for cosh, sinh and tanh. According to the separation (7) and (8) and the decomposition (9), both $\phi_{p q}^{R}$ and $\phi^{S}$ must satisfy the Laplace equation (2), the kinematic-dynamic boundary condition on the free surface (3), and the no-flux condition on the seabed (4). Let $x_{p}^{ \pm}$indicate the $x$-coordinate of the rest position of the vertical surface of the $p$ th array:

$$
x_{p}^{ \pm}=(p-1) L \pm b
$$

Each gate $G_{p q}$ spans a $y$-width given by:

$$
y \in\left[y_{q}, y_{q+1}\right], y_{q}=(q-1) a-\frac{w}{2}, \quad q=1, \ldots, Q .
$$

The kinematic boundary conditions on the gate-farm surfaces then become:

$$
\begin{aligned}
& \frac{\partial \phi_{p q}^{R}}{\partial x}= \begin{cases}-\mathrm{i} \omega \theta_{p q}(z+h), & x=x_{p}^{ \pm}, y \in\left[y_{q}, y_{q+1}\right], z \in[-h, 0], \\
0, & \text { elsewhere on the gate farm, }\end{cases} \\
& \frac{\partial \phi_{p q}^{R}}{\partial y}=0, \quad x \in\left[x_{p}^{-}, x_{p}^{+}\right], y= \pm \frac{w}{2}, z \in[-h, 0], \\
& \frac{\partial \phi^{S}}{\partial x}=-\frac{\partial \phi^{I}}{\partial x}, \quad x=x_{p}^{ \pm}, y \in\left[y_{q}, y_{q+1}\right], z \in[-h, 0] \\
& \frac{\partial \phi^{S}}{\partial y}=0, \quad x \in\left[x_{p}^{-}, x_{p}^{+}\right], y= \pm \frac{w}{2}, z \in[-h, 0] \\
& p=1, \ldots, P, \quad q=1, \ldots, Q .
\end{aligned}
$$

Finally $\phi_{p q}^{R}$ and $\phi^{S}$ must be outgoing when $\sqrt{x^{2}+y^{2}} \rightarrow \infty$.

Separation of variables gives:

$$
\left\{\begin{array}{l}
\phi_{p q}^{R} \\
\phi^{S}
\end{array}\right\}=\sum_{n=0}^{\infty}\left\{\begin{array}{l}
\varphi_{n, p q}^{R}(x, y) \\
\varphi_{n}^{S}(x, y)
\end{array}\right\} Z_{n}(z),
$$

where $Z_{n}(z)$ represents the normalized eigenfunctions:

$$
Z_{n}(z)=\frac{\sqrt{2} \operatorname{ch} k_{n}(h+z)}{\left(h+\frac{g}{\omega^{2}} \operatorname{sh}^{2} k_{n} h\right)^{1 / 2}},
$$

which satisfy the orthogonality property

$$
\int_{-h}^{0} Z_{n}(z) Z_{m}(z) d z=\delta_{n m}, \quad n, m=0,1, \ldots
$$

with $\delta_{n m}$ the Kronecker delta. In (15), $k_{n}$ are the roots of the dispersion relation:

$$
\begin{aligned}
& \omega^{2}=g k_{0} \text { th } k_{0} h, \\
& \omega^{2}=-g \bar{k}_{n} \tan \bar{k}_{n} h, \quad k_{n}=\mathrm{i} \bar{k}_{n}, n=1, \ldots, \infty .
\end{aligned}
$$


Following (14), for each of the $\varphi_{n, p q}^{R}, \varphi_{n}^{S}$, the Laplace equation becomes the Helmholtz equation

$$
\mathcal{L}\left\{\begin{array}{l}
\varphi_{n, p q}^{R}(x, y) \\
\varphi_{n}^{S}(x, y)
\end{array}\right\}=0, \quad \text { with } \mathcal{L} \equiv\left(\nabla^{2}+k_{n}^{2}\right) .
$$

Now define the boundary $S_{p q}$ of the gate $G_{p q}$ as

$$
S_{p q}=\left\{x=x_{p}^{ \pm}, y \in\left[y_{q}, y_{q+1}\right]\right\},
$$

and the end boundaries of the $p$ th array of width $2 b$

$$
S_{p}=\left\{x \in\left[x_{p}^{-}, x_{p}^{+}\right], y= \pm \frac{w}{2}\right\} \text {. }
$$

We can so refer to the entire gate farm boundary $S_{G}$ as:

$$
S_{G}=\sum_{p=1}^{P} \sum_{q=1}^{Q} S_{p q} \cup \sum_{p=1}^{P} S_{p} .
$$

The boundary conditions (13a)-(13e) become

$$
\begin{aligned}
& \frac{\partial \varphi_{n, p q}^{R}}{\partial x}= \begin{cases}-\mathrm{i} \omega \theta_{p q} f_{n}, & \text { on } S_{p q} \\
0, & \text { elsewhere }\end{cases} \\
& \frac{\partial \varphi_{n, p q}^{R}}{\partial y}=0, \quad \text { on } S_{p}, \\
& \frac{\partial \varphi_{n}^{S}}{\partial x}=A d_{n} e^{-\mathrm{i} k_{n} x}, \quad \text { on } S_{p q} \\
& \frac{\partial \varphi_{n}^{S}}{\partial y}=0, \quad \text { on } S_{p}, \\
& p=1, \ldots, P, q=1, \ldots, Q,
\end{aligned}
$$

where the coefficients $f_{n}$ and $d_{n}$ are

$$
\begin{array}{cc}
f_{n}=\frac{\sqrt{2}\left(1-\operatorname{ch} k_{n} h+k_{n} h \operatorname{sh} k_{n} h\right)}{\left(h+\frac{g}{\omega^{2}} \operatorname{sh}^{2} k_{n} h\right)^{1 / 2} k_{n}^{2}}, & n=0,1, \ldots \\
d_{n}=\frac{g k_{n}\left(h+\frac{g}{\omega^{2}} \operatorname{sh}^{2} k_{n} h\right)^{1 / 2}}{\sqrt{2} \omega \operatorname{ch} k_{n} h} \delta_{0 n}, \quad n=0,1, \ldots
\end{array}
$$

Note that in (24) only $d_{0}$ is non-zero. We also require $\varphi_{n, p q}^{R}$ and $\varphi_{n}^{S}$ to be outgoing as $\sqrt{x^{2}+y^{2}} \rightarrow \infty$. The solution of the boundary value problem defined by the Helmholtz equation (18) and by the boundary conditions (22a)-(22e) can be found by using Green's theorem and Green's functions.

Consider the plane fluid domain $\Sigma$ enclosed within the boundary of the gate farm $S_{G}$ and a circle of large radius $S_{\infty}$ surrounding the gate farm. Define the Green function $G_{n}(x, y ; \xi, \eta)$ as the solution of the Helmholtz equation:

$$
\mathcal{L} G_{n}(x, y ; \xi, \eta)=0, \quad(x, y) \in \Sigma,(x, y) \neq(\xi, \eta),
$$

with

$$
G_{n} \simeq \frac{1}{2 \pi} \ln r, \quad r \rightarrow 0
$$

where $r=\sqrt{(x-\xi)^{2}+(y-\eta)^{2}}$.

$G_{n}$ must be outgoing as $r \rightarrow \infty$, hence the solution of (25) and (26) is:

$$
G_{n}(x, y ; \xi, \eta)=-\frac{\mathrm{i}}{4} H_{0}^{(1)}\left(k_{n} r\right) .
$$

In the latter, $H_{0}^{(1)}$ is the Hankel function of the first kind and order zero.

Application of Green's theorem yields

$$
\begin{aligned}
& \iint_{\bar{\Sigma}}\left[\left\{\varphi_{n, p q}^{R}(x, y) \varphi_{n}^{S}(x, y)\right\} \mathcal{L} G_{n}(x, y ; \xi, \eta)-G_{n}(x, y ; \xi, \eta) \mathcal{L}\left\{\varphi_{n, p q}^{R}(x, y) \varphi_{n}^{S}(x, y)\right\}\right] d \Sigma \\
& ==\oint_{S_{G}+S_{\infty}+S_{\epsilon}}\left[\left\{\varphi_{n, p q}^{R}(x, y) \varphi_{n}^{S}(x, y)\right\} \frac{\partial G_{n}(x, y ; \xi, \eta)}{\partial n}-G_{n}(x, y ; \xi, \eta) \frac{\partial}{\partial n}\left\{\varphi_{n, p q}^{R}(x, y) \varphi_{n}^{S}(x, y)\right\}\right] d S
\end{aligned}
$$


where $\bar{\Sigma}=\Sigma \backslash(\xi, \eta), S_{\epsilon}$ is a semicircle of radius $\epsilon \rightarrow 0$ centred at $(\xi, \eta)$ and finally $\partial(\cdot) / \partial n$ is the derivative of $(\cdot)$ in the direction of the outward normal to the boundaries of $\bar{\Sigma}$.

Because of the governing Eqs. (18)-(25) and the behaviour of $G_{n}$ for $r \rightarrow 0$ (26) and $r \rightarrow \infty$, Eq. (28) simplifies to (see also Linton and McIver [17]-Mei et al. [25])

$$
\int_{S_{G}}\left[\left\{\begin{array}{c}
\varphi_{n, p q}^{R}(\xi, \eta) \\
\varphi_{n}^{S}(\xi, \eta)
\end{array}\right\} \frac{\partial G_{n}}{\partial n}-G_{n} \frac{\partial}{\partial n}\left\{\begin{array}{c}
\varphi_{n, p q}^{R}(\xi, \eta) \\
\varphi_{n}^{S}(\xi, \eta)
\end{array}\right\}\right] d S-\frac{1}{2}\left\{\begin{array}{c}
\varphi_{n, p q}^{R}(x, y) \\
\varphi_{n}^{S}(x, y)
\end{array}\right\}=0,(x, y) \in S_{G},
$$

where the line integral is now evaluated in terms of $(\xi, \eta)$ on the boundary $S_{G}$. The radiation potential $\varphi_{n, p q}^{R}$ and the scattering potential $\varphi_{n}^{S}$ are expressed in integral form. Define $\xi_{p}^{ \pm}$and $\eta_{q}$ as follows:

$$
\xi_{p}^{ \pm}=x_{p}^{ \pm}, \quad \eta_{q}=y_{q}
$$

Since:

$$
\frac{\partial}{\partial n}= \begin{cases}\mp \frac{\partial}{\partial \xi} & \text { on } S_{p q} \\ \mp \frac{\partial}{\partial \eta} & \text { on } S_{p}\end{cases}
$$

substitution of the boundary conditions (22a)-(22e) inside Eq. (29), yields:

$$
\begin{aligned}
\varphi_{n, p q}^{R}(x, y)= & 2 \sum_{p^{*}=1}^{P}\left\{-\left.\int_{-\frac{w}{2}}^{\frac{w}{2}} \varphi_{n, p q}^{R}(\xi, \eta) \frac{\partial G_{n}}{\partial \xi}\right|_{\xi=\xi_{p^{*}}^{+}} d \eta+\left.\int_{-\frac{w}{2}}^{\frac{w}{2}} \varphi_{n, p q}^{R}(\xi, \eta) \frac{\partial G_{n}}{\partial \xi}\right|_{\xi=\xi_{p^{*}}^{-}} d \eta-\left.\int_{\xi_{p^{*}}^{-}}^{\xi_{p^{*}}^{+}} \varphi_{n, p q}^{R}(\xi, \eta) \frac{\partial G_{n}}{\partial \eta}\right|_{\eta=\frac{w}{2}} d \xi\right. \\
& \left.+\left.\int_{\xi_{p^{*}}^{-}}^{\xi_{p^{*}}^{+}} \varphi_{n, p q}^{R}(\xi, \eta) \frac{\partial G_{n}}{\partial \eta}\right|_{\eta=-\frac{w}{2}} d \xi\right\}+2 \mathrm{i} \omega \theta_{p q} f_{n} \int_{\eta_{q}}^{\eta_{q+1}}\left(\left.G_{n}\right|_{\xi=\xi_{p}^{-}}-\left.G_{n}\right|_{\xi=\xi_{p}^{+}}\right) d \eta, \quad(x, y) \in S_{G}, \\
\varphi_{n}^{S}(x, y)= & 2 \sum_{p^{*}=1}^{P}\left\{-\left.\int_{-\frac{w}{2}}^{\frac{w}{2}} \varphi_{n}^{S}(\xi, \eta) \frac{\partial G_{n}}{\partial \xi}\right|_{\xi=\xi_{p^{*}}^{+}} d \eta+\left.\int_{-\frac{w}{2}}^{\frac{w}{2}} \varphi_{n}^{S}(\xi, \eta) \frac{\partial G_{n}}{\partial \xi}\right|_{\xi=\xi_{p^{*}}} d \eta-\left.\int_{\xi_{p^{*}}^{-}}^{\xi_{p^{*}}^{+}} \varphi_{n}^{S}(\xi, \eta) \frac{\partial G_{n}}{\partial \eta}\right|_{\eta=\frac{w}{2}} d \xi\right. \\
& \left.+\left.\int_{\xi_{p^{*}}^{-}}^{\xi_{p^{*}}^{+}} \varphi_{n}^{S}(\xi, \eta) \frac{\partial G_{n}}{\partial \eta}\right|_{\eta=-\frac{w}{2}} d \xi+A d_{n} \int_{-\frac{w}{2}}^{\frac{w}{2}}\left(\left.e^{-i k_{n} \xi} G_{n}\right|_{\xi=\xi_{p^{*}}^{+}}-\left.e^{-\mathrm{i} k_{n} \xi} G_{n}\right|_{\xi=\xi_{p^{*}}^{-}}\right) d \eta\right\}, \quad(x, y) \in S_{G} .
\end{aligned}
$$

Note that (32) and (33) are more complex than their thin-gate counterparts of Renzi et al. [14]. Since the radiation potential $\varphi_{n, p q}^{R}$ and the scattering potential $\varphi_{n}^{S}$ on the boundary of the gate-farm are unknown, the first four integrals inside the expressions (32) and (33) are still unknown. The integrals inside the summations are evaluated on the boundary of each array, except for the last integral of (32) which is evaluated on the boundary of the moving gate $G_{p q}$. Imposing the boundary conditions (22a)-(22e) to (32) and (33) yields a system of hypersingular integral equations for $\varphi_{n, p q}^{R}$ and $\varphi_{n}^{S}$ evaluated on the boundaries of the gate farm. The solution of the system is found by expanding $\varphi_{n, p q}^{R}$ and $\varphi_{n}^{S}$ in terms of Legendre polynomials $P_{m}$ of integer order $m=0, \ldots, M$ (see Appendix for details). Finally the radiation potential $\phi_{p q}^{R}$ due to the motion of the gate $G_{p q}$, on the lateral surfaces of each array $\tilde{p}=1, \ldots, P$, is expressed as follow:

$$
\left\{\begin{array}{l}
\phi_{p q}^{R}\left(x_{\tilde{p}}^{ \pm}, y, z\right) \\
\phi_{p q}^{R}\left(x, \pm \frac{w}{2}, z\right)
\end{array}\right\}=\sum_{n=0}^{\infty} \sum_{m=0}^{M} Z_{n}(z) \theta_{p q}\left\{\begin{array}{c}
P_{m}\left(y^{\prime}\right) \alpha_{n m \tilde{p}, p q}^{R \pm} \\
P_{m}\left(x_{\tilde{p}}^{\prime}\right) \beta_{n m \tilde{p}, p q}^{R \pm}
\end{array}\right\},
$$

while the scattering potential on the same surfaces is given by:

$$
\begin{aligned}
& \left\{\begin{array}{l}
\phi^{S}\left(x_{\tilde{p}}^{ \pm}, y, z\right) \\
\phi^{S}\left(x, \pm \frac{w}{2}, z\right)
\end{array}\right\}=\sum_{m=0}^{M} Z_{0}(z)\left\{\begin{array}{l}
P_{m}\left(y^{\prime}\right) \alpha_{0 m \tilde{p}}^{S \pm} \\
P_{m}\left(x_{\tilde{p}}^{\prime}\right) \beta_{0 m \tilde{p}}^{S \pm}
\end{array}\right\}, \\
& x \in[-b+(\tilde{p}-1) L, b+(\tilde{p}-1) L], y \in\left[-\frac{w}{2}, \frac{w}{2}\right],
\end{aligned}
$$

where $x_{\tilde{p}}^{\prime}$ and $y^{\prime}$ are dimensionless variables defined in $[-1,1]$ :

$$
x_{\tilde{p}}^{\prime}=\frac{x-(\tilde{p}-1) L}{b}, y^{\prime}=\frac{2 y}{w}
$$


while $\alpha_{n m \tilde{p}, p q}^{R \pm}, \alpha_{0 m \tilde{p}}^{S \pm}, \beta_{n m \tilde{p}, p q}^{R \pm}$ and $\beta_{0 m \tilde{p}}^{S \pm}$ are complex constants determined by solving the linear systems (A.38a)-(A.38c) and (A.39a) and (A.39b) with a numerical collocation scheme (see Appendix for further details).

\subsection{Gate dynamics}

Consider each gate $G_{p q}$ coupled with an energy generator at the hinge. Assume that the generator exerts a torque proportional to the angular velocity of the gate $G_{p q}, v_{p t o} \dot{\Theta}_{p q}$, where $v_{p t o}$ is the power take-off coefficient. Conservation of angular momentum requires:

$$
I \ddot{\Theta}_{p q}+C \Theta_{p q}+v_{p t o} \dot{\Theta}_{p q}=\rho \int_{y_{q}}^{y_{q+1}} d y \int_{-h}^{0}\left[\left.\Phi\right|_{x=x_{p}^{+}}-\left.\Phi\right|_{x=x_{p}^{-}}\right]_{t}(z+h) d z
$$

where $I$ is the moment of inertia of the gate about the hinge and $C$ is the net restoring torque:

$$
C=\rho g\left(I_{x x}^{A}+I_{z}^{V}\right)-M_{g} g\left(z_{g}+h\right),
$$

with:

$$
I_{x x}^{A}=\iint_{S_{A}} x^{2} d x d y, \quad I_{z}^{V}=\iiint_{V}(z+h) d V,
$$

where $S_{A}$ denotes the cross sectional area of the gate at the water line and $V$ the water volume displaced by the gate in its rest vertical position. $M_{g}$ and $z_{g}$ are respectively the mass and the vertical coordinate of the center of mass of the gate. For the geometry of Fig. $1, I_{x x}^{A}$ and $I_{z}^{V}$ are:

$$
I_{x x}^{A}=\frac{2 a b^{3}}{3}, I_{z}^{V}=a b h^{2} .
$$

Using (7)-(9) and the expressions of the potentials (10), (34) and (35), the momentum equation (37) gives

$$
\left(-\omega^{2} I+C-\mathrm{i} \omega v_{p t o}\right) \theta_{p q}-\sum_{\bar{p}=1}^{P} \sum_{\bar{q}=1}^{Q} \theta_{\overline{p q}}\left(\omega^{2} \mu_{\overline{p q}}^{p q}+\mathrm{i} \omega \nu_{\overline{p q}}^{p q}\right)=F_{p q}, \quad p=1, \ldots, P ; \quad q=1, \ldots Q,
$$

where

$$
F_{p q}=-\mathrm{i} \omega \rho\left\{\frac{2 A g a e^{-i k_{0}(p-1) L} \sin k_{0} b\left(1-\operatorname{ch} k_{0} h+k_{0} h \operatorname{sh} k_{0} h\right)}{\omega k_{0}^{2} \operatorname{ch} k_{0} h}+f_{0} \int_{y_{q}}^{y_{q+1}} \sum_{m=0}^{\infty}\left(\alpha_{0 m p}^{+S}-\alpha_{0 m p}^{S-}\right) P_{m}\left(\frac{2 y}{w}\right) d y\right\},
$$

is the exciting torque due to the incident and scattered waves, while:

$$
\mu \frac{p q}{p q}=\frac{\rho}{\omega} \operatorname{Im}\left\{\sum_{n=0}^{\infty} f_{n} \int_{y_{q}}^{y_{q+1}} \sum_{m=0}^{M}\left(\alpha_{n m p, \overline{p q}}^{R+}-\alpha_{n m p, \overline{p q}}^{R-}\right) P_{m}\left(\frac{2 y}{w}\right) d y\right\},
$$

and

$$
\nu_{\overline{p q}}^{p q}=-\rho \operatorname{Re}\left\{\sum_{n=0}^{\infty} f_{n} \int_{y_{q}}^{y_{q+1}} \sum_{m=0}^{M}\left(\alpha_{n m p, \overline{p q}}^{R+}-\alpha_{n m p, \overline{p q}}^{R-}\right) P_{m}\left(\frac{2 y}{w}\right) d y\right\},
$$

represent, respectively, the added inertia and the radiation damping of the gate $G_{p q}$ due to the unit rotation of the gate $G_{\overline{p q}}$. Eq. (41) can be written in matrix form:

$$
\left[\left(-\omega^{2} I+C-\mathrm{i} \omega v_{p t o}\right) \mathbf{I}-\omega^{2} \mathbf{M}(\omega)-\mathrm{i} \omega \mathbf{N}(\omega)\right]\{\theta\}=\mathbf{F}(\omega)
$$

where $\{\theta\}$ is a column vector of length $s=P \times Q$ that contains all the angular displacements of the gates:

$$
\{\theta\}=\left\{\begin{array}{l}
\left\{\theta_{1}\right\} \\
\vdots \\
\left\{\theta_{p}\right\} \\
\vdots \\
\left\{\theta_{P}\right\}
\end{array}\right\},
$$

I is the identity matrix of size $s \times s, \mathbf{M}$ and $\mathbf{N}$ are respectively the added inertia matrix and the radiation damping matrix also of size $s \times s$ :

$$
\mathbf{M}=\left[\begin{array}{lll}
\mathbf{M}_{1}^{1} & \ldots & \mathbf{M}_{P}^{1} \\
\vdots & \ddots & \vdots \\
\mathbf{M}_{1}^{P} & \ldots & \mathbf{M}_{P}^{P}
\end{array}\right], \mathbf{N}=\left[\begin{array}{lll}
\mathbf{N}_{1}^{1} & \ldots & \mathbf{N}_{P}^{1} \\
\vdots & \ddots & \vdots \\
\mathbf{N}_{1}^{P} & \ldots & \mathbf{N}_{P}^{P}
\end{array}\right]
$$


where both $\mathbf{M}_{m}^{m}$ and $\mathbf{N}_{p}^{p}$ are symmetrical square matrices of size $Q \times Q$ :

$$
\mathbf{M}_{\bar{p}}^{p}=\left[\begin{array}{lll}
\mu_{\bar{p} 1}^{p 1} & \ldots & \mu_{\bar{p} Q}^{p 1} \\
\vdots & \ddots & \vdots \\
\mu_{\bar{p} 1}^{p Q} & \ldots & \mu_{\bar{p} Q}^{p Q}
\end{array}\right], \mathbf{N}_{\bar{p}}^{p}=\left[\begin{array}{lll}
\nu_{\bar{p} 1}^{p 1} & \ldots & v_{\bar{p} Q}^{p 1} \\
\vdots & \ddots & \vdots \\
v_{\bar{p} 1}^{p Q} & \ldots & v_{\bar{p} Q}^{p Q}
\end{array}\right] .
$$

Finally, once the angular displacements of the gates are known, the average power absorbed over a wave cycle by the gate farm, is equal to:

$$
P=\frac{\omega^{2} v_{p t o}}{2} \sum_{p=1}^{P} \sum_{q=1}^{Q}\left|\theta_{p q}\right|^{2}
$$

\subsection{Eigenfrequencies and eigenvectors}

The momentum equations given by (45) are equivalent to a system of $P \times Q$ linear damped harmonic oscillators with given mass, stiffness and damping. In order to find the eigenfrequencies of the system, the exciting torque and the damping terms are set equal to zero. System (45) becomes homogeneous:

$$
\left[\left(-\omega^{2} I+C\right) \mathbf{I}-\omega^{2} \mathbf{M}(\omega)\right]\{\theta\}=0 .
$$

To find non-trivial solutions the following implicit non linear eigenvalue condition must then be solved:

$$
\operatorname{det}\left[\left(-\omega^{2} I+C\right) \mathbf{I}-\omega^{2} \mathbf{M}(\omega)\right]=0
$$

Once the eigenfrequencies are known, the respective modal forms can be obtained by setting the displacement of the gate $G_{11}=1$ and then solving system (50).

\subsection{The radiation potential in the far field}

Consider the polar coordinates $r$ and $\gamma$ defined by

$$
(x, y)=r(\cos \gamma, \sin \gamma) .
$$

Following a similar procedure as in Renzi and Dias [10], the radiation potential in the far field (i.e. for $r \rightarrow \infty$ ), for unit rotational velocity of the gate $G_{p q}$, can be approximated as

$$
\phi_{p q}^{R}(r, \gamma, z) \simeq \frac{-i g \mathcal{A}_{p q}^{R}(\gamma) \operatorname{ch} k(h+z)}{\omega \operatorname{ch} k h} \sqrt{\frac{2}{\pi k r}} e^{i k r-\frac{i \pi}{4}},
$$

where

$$
\begin{aligned}
\mathcal{A}_{p q}^{R}(\gamma)= & -\frac{k Z(0)}{4 g} \sum_{p^{*}=1}^{P} \sum_{m=0}^{M}\left\{\int_{-\frac{w}{2}}^{\frac{w}{2}} \alpha_{0 m p^{*}, p q}^{R+} P_{m}\left(\eta^{\prime}\right) e^{-i k\left\{\left[b+\left(p^{*}-1\right) L\right] \cos \gamma+\eta \sin \gamma\right\}} \cos \gamma d \eta\right. \\
& -\int_{-\frac{w}{2}}^{\frac{w}{2}} \alpha_{0 m p^{*}, p q}^{R-} P_{m}\left(\eta^{\prime}\right) e^{-i k\left\{\left[-b+\left(p^{*}-1\right) L\right] \cos \gamma+\eta \sin \gamma\right\}} \cos \gamma d \eta+\int_{\xi_{p^{*}}^{-}}^{\xi_{p^{*}}^{+}} \beta_{0 m p^{*}, p q}^{R+} P_{m}\left(\xi_{p^{*}}^{\prime}\right) e^{-i k\left[\xi \cos \gamma+\frac{w}{2} \sin \gamma\right]} \sin \gamma d \xi \\
& \left.-\int_{\xi_{p^{*}}^{-}}^{\xi_{p^{*}}^{+}} \beta_{0 m p^{*}, p q}^{R-} P_{m}\left(\xi_{p^{*}}^{\prime}\right) e^{-i k\left[\xi \cos \gamma-\frac{w}{2} \sin \gamma\right]} \sin \gamma d \xi\right\} \\
& -\frac{\omega f_{n} Z(0)}{4 g} \int_{\eta_{q}}^{\eta_{q+1}}\left(e^{-i k\{[-b+(p-1) L] \cos \gamma+\eta \sin \gamma\}}-e^{-i k\{[b+(p-1) L] \cos \gamma+\eta \sin \gamma\}}\right) d \eta,
\end{aligned}
$$

represents the angular variation of the radially spreading wave (Mei et al. [25]). The latter can be used to derive some useful formulas that relate the hydrodynamic parameters.

\subsection{The Haskind-Hanaoka relation for the gate farm}

Consider the 3D Haskind-Hanaoka relation (Mei et al. [25])

$$
F_{p q}=-\frac{4}{k} \rho g A \mathcal{A}_{p q}^{R}(0) C_{g}
$$


Table 1

Gate farm characteristics.

\begin{tabular}{lll}
\hline Parameters & Symbol & Value \\
\hline Gate width & $a$ & $3 \mathrm{~m}$ \\
Gate thickness & $2 b$ & $1.5 \mathrm{~m}$ \\
Distance between arrays & $L$ & $10 \mathrm{~m}$ \\
Moment of inertia & $I$ & $72,000 \mathrm{~kg} \mathrm{~m}^{2}$ \\
Buoyancy restoring torque & $C$ & $300,000 \mathrm{~kg} \mathrm{~m}^{2} \mathrm{~s}^{-2}$ \\
Gate mass & $M_{g}$ & $2600 \mathrm{~kg}^{-3}$ \\
Water depth & $h$ & $5 \mathrm{~m}$ \\
Density of water & $\rho$ & $1000 \mathrm{~kg} \mathrm{~m}^{-3}$ \\
\hline
\end{tabular}

Table 2

Eigenfrequency $\omega_{1}$ of the single gate in the open sea for different values of $2 b$.

\begin{tabular}{lll}
\hline $2 b(\mathrm{~m})$ & $\omega_{1}(\mathrm{rad} / \mathrm{s})$ & Period $(\mathrm{s})$ \\
\hline 0.1 & 0.89 & 7.05 \\
0.45 & 0.86 & 7.30 \\
0.8 & 0.84 & 7.47 \\
1.15 & 0.82 & 7.65 \\
1.5 & 0.81 & 7.75 \\
\hline
\end{tabular}

where $F_{p q}$ is the exciting torque given by expression (42) while $\mathcal{A}_{p q}^{R}(0)$ represents the wave amplitude in the direction opposite to the incident waves

$$
\mathcal{A}_{p q}^{R}(0)=-\frac{a k Z(0)}{2 g} \sum_{p^{*}=1}^{P}\left\{\alpha_{00 p^{*}, p q}^{R+} e^{-i k\left[b+\left(p^{*}-1\right) L\right]}-\alpha_{00 p^{*}, p q}^{R-} e^{-i k\left[-b+\left(p^{*}-1\right) L\right]}\right\}-\frac{\omega a f_{n} Z(0)}{2 g Q}\left(e^{-i k[-b+(p-1) L]}-e^{-i k[b+(p-1) L]}\right) .
$$

Expression (55) has been used to check the numerical computation via the relative error $\epsilon$

$$
\epsilon=\frac{\mid \text { l.h.s. }- \text { r.h.s. } \mid}{\mid \text { r.h.s. } \mid}
$$

where 1.h.s. and r.h.s. refer to Eq. (55) itself. Taking $M=16$ in (34) and (35) we obtain a maximum relative error $\epsilon=O\left(10^{-3}\right.$ ) for expression (55).

\section{Results and discussion}

\subsection{One gate in the open sea: the effects of the gate thickness}

In order to evaluate the effects of the finite gate thickness $2 b$, the simplest case of $P=Q=1$, i.e. the case of one gate in the open sea is considered. Inertia, buoyancy and width of the gate, and water depth, are listed in Table 1 . Different values of the thickness $2 b$ have been chosen, i.e. $2 b \in[0.1 ; 1.5] \mathrm{m}$. The limit value of $2 b=0.1 \mathrm{~m}$ corresponds to the case where the "thin-gate" hypothesis can be applied $(b / a \ll 1$ - Renzi and Dias [9]). Fig. 2 shows the values of the added inertia $\mu$, the radiation damping $v$ and the magnitude of the exciting torque $|F|$ versus the frequency of the incident waves for different values of $b$. The effects of the gate thickness on the added inertia and radiation damping are significant for $\omega \in[1,3.5] \mathrm{rad} \mathrm{s}^{-1}$. In particular, the larger the gate thickness the larger the added mass and radiation damping. As a consequence the eigenfrequency of the system decreases if the gate thickness increases. The eigenfrequency $\omega_{1}$ of the single gate for five different values of $2 b$ is listed in Table 2.

\subsection{The gate farm in the open sea}

With reference to Fig. 1 , we consider $P=3$ arrays each with $Q=5$ gates. The input parameters are defined in Table 1 .

\subsubsection{Eigenfrequencies and eigenvectors}

The eigenvalue condition (51) has been solved in order to find the eigenfrequencies of the system within a range of $\omega$ from 0 to $1.2 \mathrm{rad} \mathrm{s}^{-1}$. The frequency range includes the $P \times(Q-1)=12$ eigenfrequencies of the out-of-phase motion and the first two eigenfrequencies of the in-phase motion, where the pth array moves at unison. The numerical values of the eigenfrequencies are listed in Table 3 for the out-of-phase motion and in Table 4 for the in-phase motion. Solution of the momentum equations (50) gives the corresponding modal forms. Note that the generic out-of-phase natural mode $N_{i j}$ follows the same definition of Sammarco et al. [6], that is: for modes $N_{11}, N_{21}, N_{31}$, and $N_{41}$, each array has the same modal shape, but for the central array $(p=2) ;$ modes $N_{12}, N_{22}, N_{32}$, and $N_{42}$, are characterized by having the middle array $(p=2)$ with null angular displacement, while the last array $(p=3)$ is in opposite phase with respect to the first $(p=1)$; for the remaining modes $N_{13}, N_{23}, N_{33}$, and $N_{43}$, modal deformation is the same, but for the middle array $(p=2)$, which is in opposition of phase with the other two. $N\left(\omega_{1}\right)$ represents the in-phase natural mode characterized by the middle array in opposite phase with respect to the first and the last array. Similarly $N\left(\omega_{2}\right)$ represents the in-phase natural mode characterized by the middle array $(p=2)$ with null angular displacements while the arrays $p=1$ and $p=3$ are in opposition of phase. Let $K$ be 

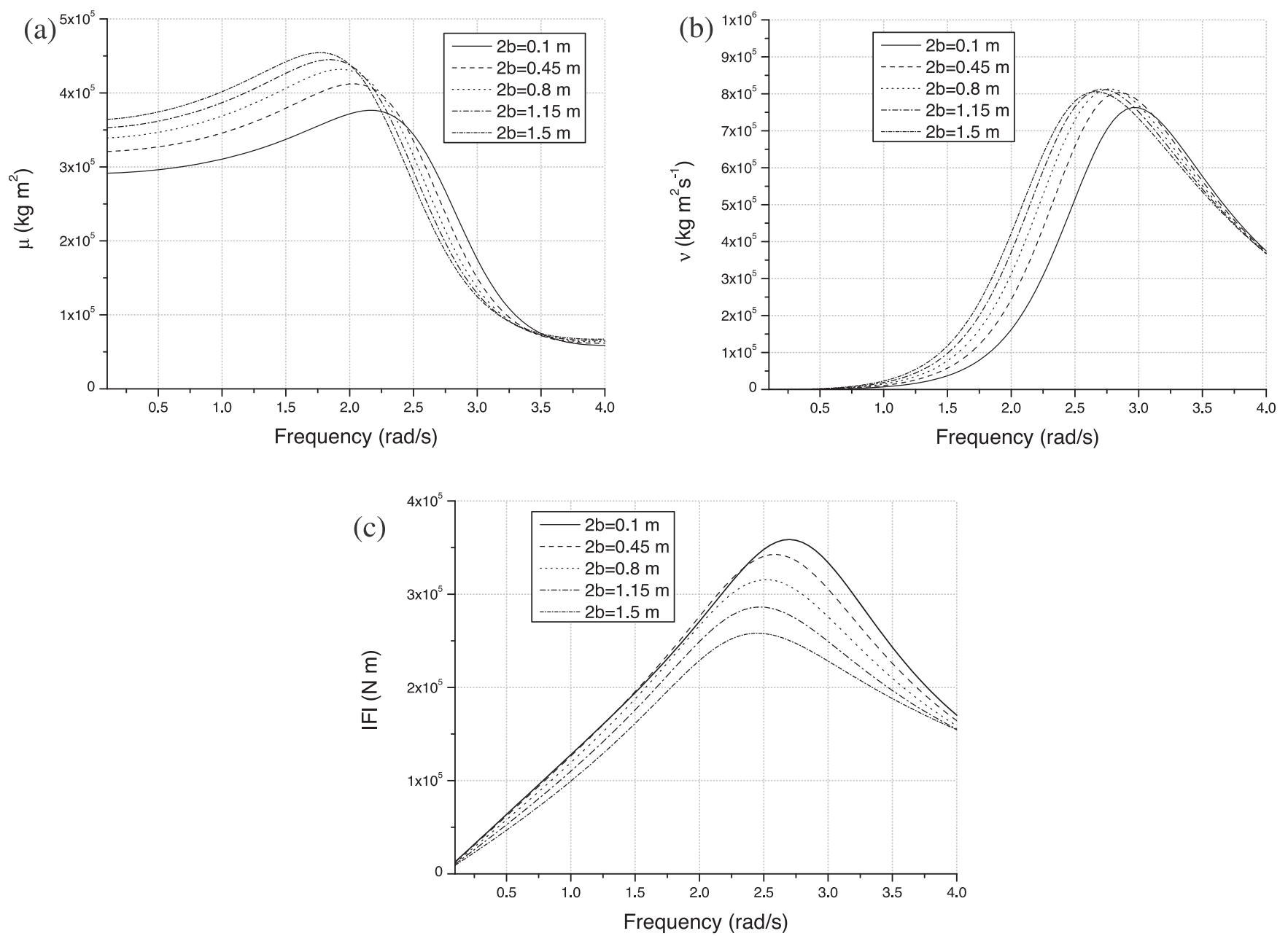

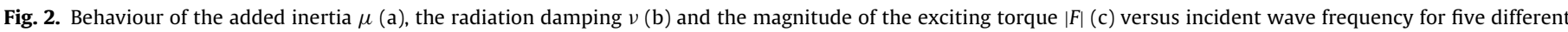
values of the gate thickness $2 b$.

Table 3

Natural frequencies of the out-of-phase modes. Note that $3 . \overline{3}$ represents the number $3.333 \ldots$

\begin{tabular}{lcll}
\hline$\omega(\mathrm{rad} / \mathrm{s})$ & Period $(\mathrm{s})$ & $K$ & Mode \\
\hline 1.013 & 6.199 & 2.5 & $N_{11}$ \\
1.012 & 6.205 & 2.5 & $N_{12}$ \\
1.011 & 6.211 & 2.5 & $N_{13}$ \\
0.934 & 6.723 & $3 . \overline{3}$ & $N_{21}$ \\
0.931 & 6.745 & $3 . \overline{3}$ & $N_{22}$ \\
0.929 & 6.760 & $3 . \overline{3}$ & $N_{23}$ \\
0.814 & 7.715 & 5 & $N_{31}$ \\
0.805 & 7.801 & 5 & $N_{32}$ \\
0.793 & 7.919 & 5 & $N_{33}$ \\
0.679 & 9.248 & 10 & $N_{41}$ \\
0.644 & 9.751 & 10 & $N_{42}$ \\
\end{tabular}

Table 4

Natural frequencies of the in-phase modes.

\begin{tabular}{lll}
\hline$\omega(\mathrm{rad} / \mathrm{s})$ & Period $(\mathrm{s})$ & Mode \\
\hline 0.395 & 15.898 & $N\left(\omega_{2}\right)$ \\
0.366 & 17.158 & $N\left(\omega_{1}\right)$ \\
\hline
\end{tabular}


Table 5

$\operatorname{Re}\left\{\theta_{11}\right\}$ at the resonance frequencies.

\begin{tabular}{ll}
\hline $\operatorname{Re}\left\{\theta_{11}\right\}(\mathrm{rad})$ & Mode \\
\hline 5.18 & $N_{11}$ \\
-3.3 & $N_{12}$ \\
-4.02 & $N_{13}$ \\
1.83 & $N_{31}$ \\
-7.98 & $N_{32}$ \\
-14.11 & $N_{33}$ \\
-9.04 & $N\left(\omega_{1}\right)$ \\
-9.68 & $N\left(\omega_{2}\right)$ \\
& \\
\hline
\end{tabular}

the number of the gates per modal wavelength of the first array, $p=1$; the eigenfrequencies of the out-of-phase modes decrease as $K$ increases.

\subsubsection{Irregular frequencies}

Because of the geometry of the gate farm, the integral equations (32) and (33) possess the so-called irregular frequencies when $n=0$ (Linton and McIver [17]-Mei et al. [25]).

Define the boundaries of the $p$ th array as

$$
S_{p}^{\prime}=\sum_{q=1}^{Q} S_{p q} \cup S_{p},
$$

and let $\Sigma_{p}^{\prime}$ be the interior of $S_{p}^{\prime}$. We can so define $\varphi_{p}^{\prime}$ as the interior potential that satisfy the Helmholtz equation in $\Sigma_{p}^{\prime}$

$$
\nabla^{2} \varphi_{p}^{\prime}+k^{2} \varphi_{p}^{\prime}=0 \text { in } \Sigma_{p}^{\prime}
$$

with boundary conditions

$$
\varphi_{p}^{\prime}=0 \text { on } S_{p}^{\prime} \text {. }
$$

The eigensolutions of the homogeneous Dirichlet problem (59) and (60) are found by separation of variables:

$$
\varphi_{p}^{\prime}=A_{n m} \sin \frac{n \pi[x-(p-1) L]}{b} \sin \frac{2 m \pi y}{w},
$$

where $A_{n m}$ is an arbitrary constant and $n, m=0,1, \ldots$.

The corresponding eigenvalues are

$$
k=k_{n m}=\sqrt{\left(\frac{n \pi}{b}\right)^{2}+\left(\frac{2 m \pi}{w}\right)^{2}},
$$

while the related eigenfrequencies $\omega_{n m}$ can be found via the dispersion relation

$$
\omega_{n m}^{2}=g k_{n m} \text { th } k_{n m} h .
$$

These eigenfrequencies are the so-called irregular frequencies (Linton and McIver [17]-Mei et al. [25]).

The lowest value of $\omega_{n m}$ corresponds to the case of $n=0$ and $m=1$ and it is equal to $\sim 2 \mathrm{rad} \mathrm{s}^{-1}$, i.e. higher than the range of our interest. For this reason we do not need to exclude them from the analysis.

\subsubsection{Forced response}

Extensive computations have been carried out for the range of interest of the incident wave frequencies $\omega=0.1-1.2$ rad s ${ }^{-1}$ without the PTO. The amplitude of the incident wave is $A=1 \mathrm{~m}$. Resonance occurs at eight frequencies whose values are near the natural frequencies of the homogeneous system previously calculated. Because of the direction of the incident wave, orthogonal to the axes of the arrays, only the symmetric natural modes with respect to the $x$-axis can be excited; i.e, $P \times(Q-1) / 2=6$ out-of-phase and 2 in-phase natural modes are resonated. Let $\omega_{i j}$ be the eigenfrequency of the out-of-phase mode $N_{i j}$. In Fig. 3 we show the amplitude of the angular displacements versus the incident wave frequency and indicate the eigenfrequencies of the resonating natural modes. Note that the high and unrealistic values of the peaks are related to the weakness of the radiation damping corresponding to the resonance frequencies. In this case the gate-farm is almost undamped and radiates low energy at infinity. Fig. 4 and Fig. 5 the shapes of the gate-farm forced at the resonance frequencies $\omega_{i j}$ are shown. Note that the number near each gate $G_{p q}$ represents $\operatorname{Re}\left\{\theta_{p q}\right\}$ normalized with respect to $\operatorname{Re}\left\{\theta_{11}\right\}$. The values of $\operatorname{Re}\left\{\theta_{11}\right\}$ at the resonance frequencies are listed in Table 5.

\subsection{The influence of the power take-off on the capture width}

A parametric analysis is performed to investigate the effect of the power take-off coefficient $v_{\text {pto }}$ on the generated power $P$ over a wave cycle (see (49)). Define the capture width ratio $C_{F}$ as the ratio of the generated power $P$ per unit gate-farm width to the incident power per unit width of the crest (see Renzi et al. [15]):

$$
C_{F}=\frac{P}{\frac{1}{2} \rho g s A^{2} C_{g} a}
$$



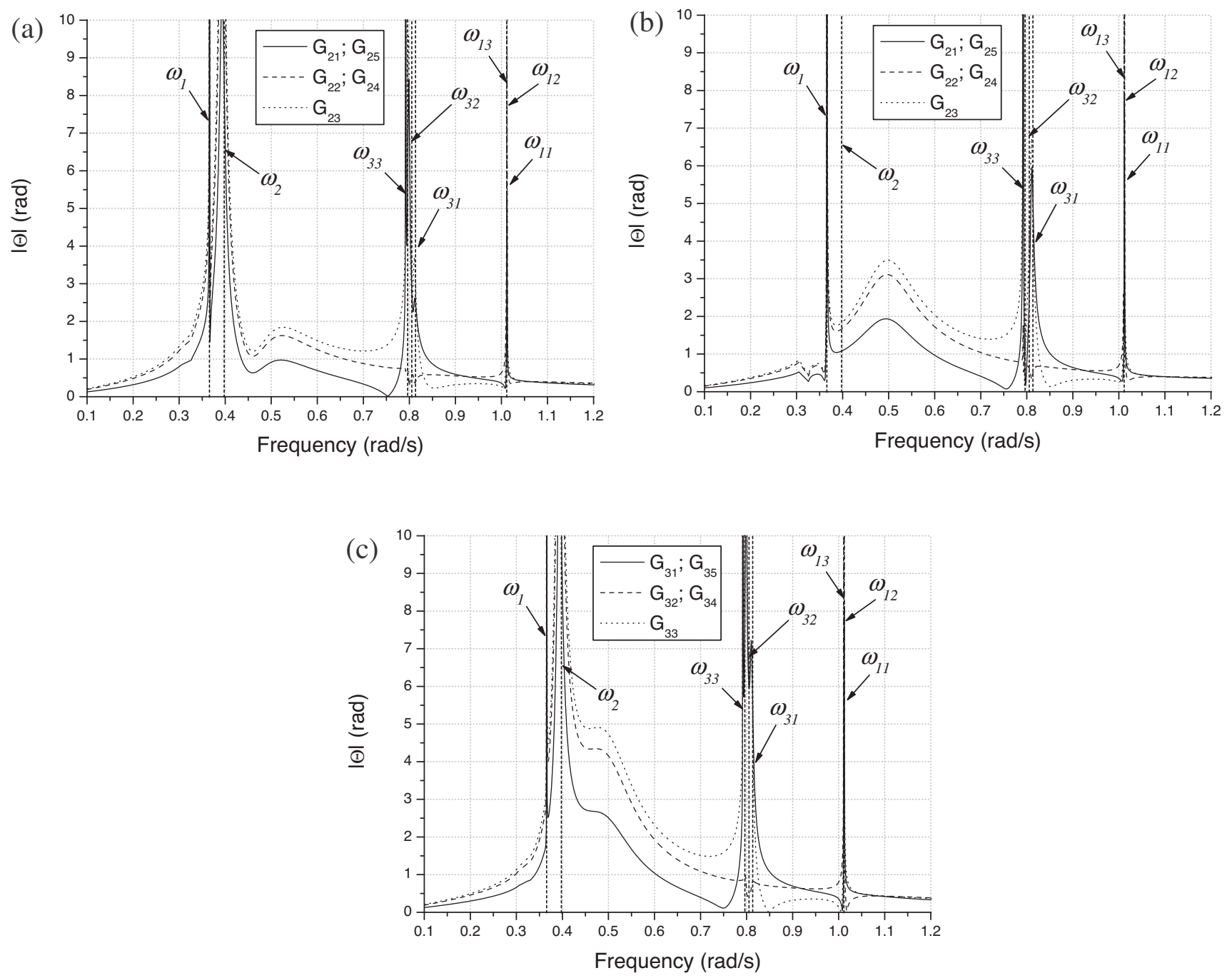

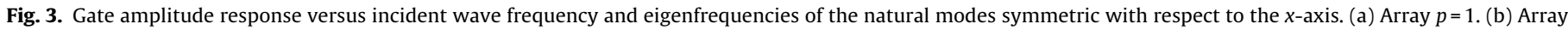
$p=2$. (c) Array $p=3$.

where $C_{g}$ is the group velocity:

$$
C_{g}=\frac{\omega}{2 k}\left(1+\frac{2 k h}{\operatorname{sh} 2 k h}\right) .
$$

Waves of amplitude $A=1 \mathrm{~m}$ are normally incident on the flaps. Different values of the PTO coefficient have been chosen, i.e. $v_{p t o} \in$ $\left[10^{4} ; 10^{8}\right] \mathrm{kg} \mathrm{m}^{2} \mathrm{~s}^{-1}$. Fig. 6 shows the behaviour of the capture width ratio $C_{F}$ versus the incident wave frequency for three different values of the PTO coefficient. When $v_{\text {pto }}=10^{6} \mathrm{~kg} \mathrm{~m}^{2} \mathrm{~s}^{-1}$ and $\omega>0.6 \mathrm{rad} \mathrm{s}^{-1}$, the capture width ratio is equal to $\sim 0.5$ for a wide range of frequencies. Consider the case of $v_{p t o}=10^{8} \mathrm{~kg} \mathrm{~m}^{2} \mathrm{~s}^{-1}$ and the behaviour of the magnitude of the exciting torque $\left|F_{p 3}\right|$ on each gate $G_{p 3}$ shown in Fig. 7: the behaviour of $C_{F}$ is quite similar to $\left|F_{p 3}\right|$. In other words, the dynamics is dominated by the exciting torque due to diffracted waves (see Renzi and Dias [10]). Differently, the behaviour of the capture width ratio for $v_{p t o}=10^{4} \mathrm{~kg} \mathrm{~m} \mathrm{~m}^{-1}$, resembles that of the amplitude of the angular displacements shown in Fig. 3, hence in this case the dynamics is dominated by the resonance effects.

\subsection{Wave power generation and efficiency: $(\mathrm{P} \times \mathrm{Q})$ gate farm versus $(\mathrm{P} \times \mathrm{Q})$ isolated gates}

In this section the $(P \times Q)$ gate farm and a system of $(P \times Q)$ isolated and independent gates are compared in terms of energy production. The single flap gate has the same characteristics for both systems (see Table 1 for the values).

Consider the PTO coefficient that maximize the power output for incident wave frequency $\omega=0.9 \mathrm{rad} \mathrm{s}^{-1}$, i.e. a typical value in the Mediterranean Sea. The optimal PTO coefficient for a system of isolated gates $v_{p t o, I G}$ can be designed such that (Renzi and Dias [10])

$$
v_{p t o, I G}=\sqrt{\frac{\left[C-(I+\mu) \omega^{2}\right]^{2}}{\omega^{2}}+v^{2}} \simeq 10^{5} \mathrm{~kg} \mathrm{~m}^{2} \mathrm{~s}^{-1},
$$



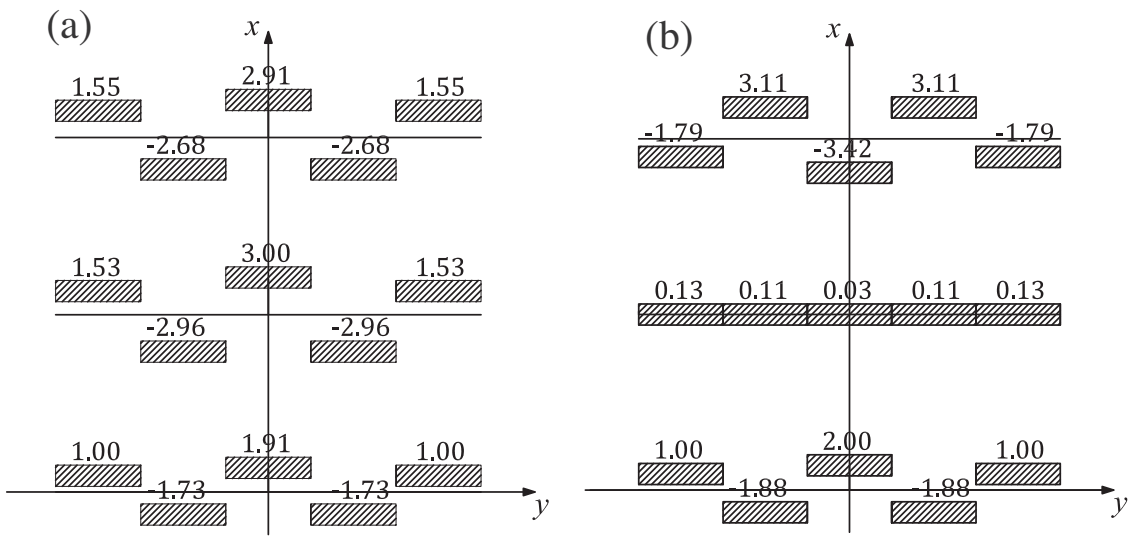

(d)
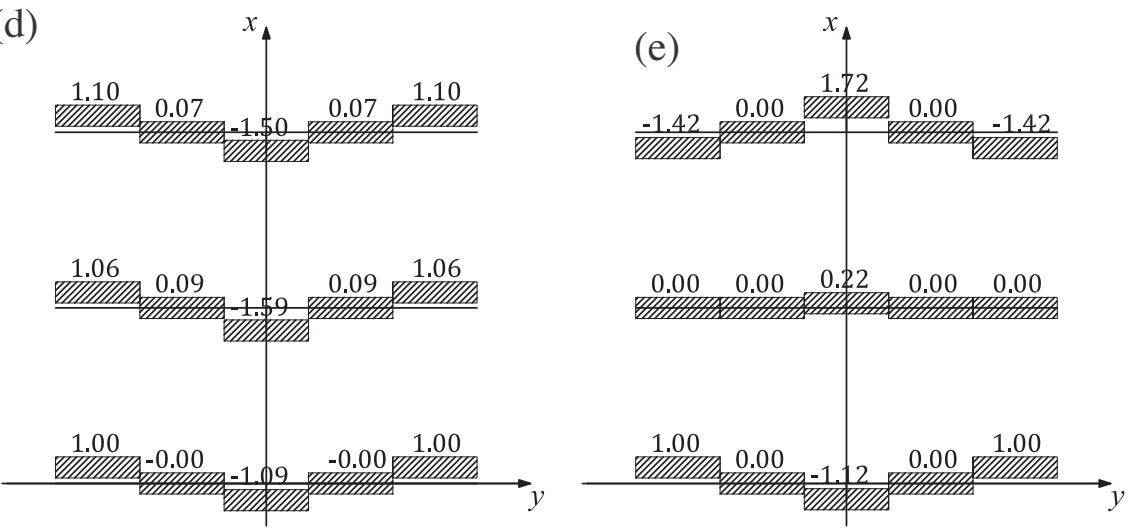
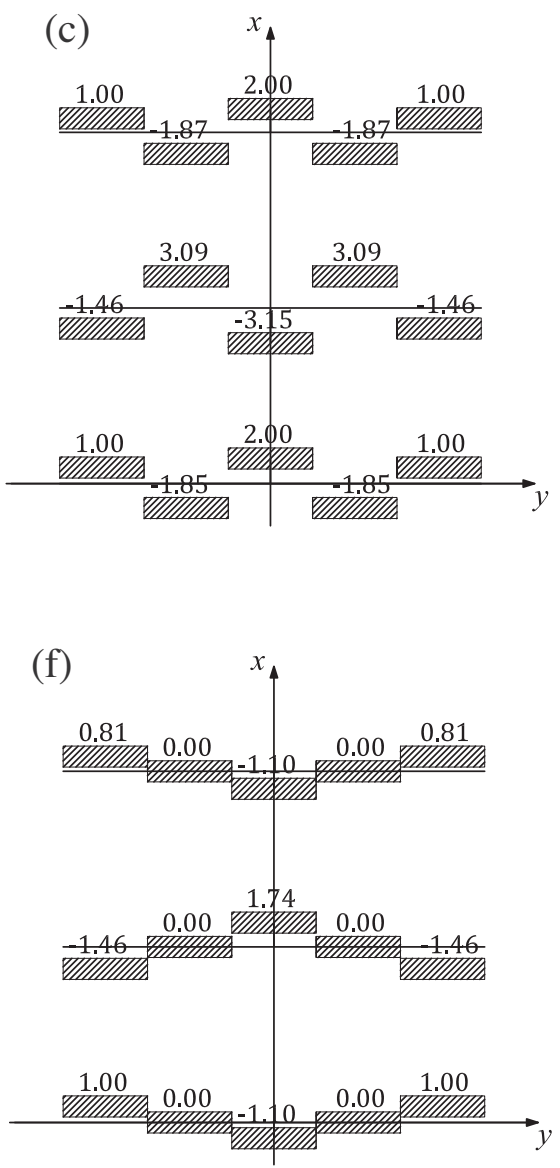

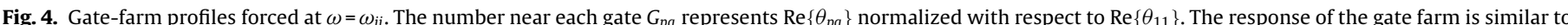

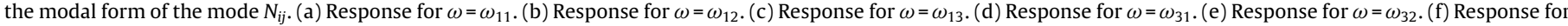
$\omega=\omega_{33}$.

where $\mu$ and $v$ represent respectively the added inertia and the radiation damping of a single isolated gate at $\omega=0.9$ rad $\mathrm{s}^{-1}$ (see Fig. 2 for the values). The optimal PTO coefficient for the gate farm $v_{p t o, G F}$ is found numerically by maximizing the function (49) for a fixed $\omega$. For $\omega=0.9 \mathrm{rad} \mathrm{s}^{-1}, v_{p t o, G F}=7 \times 10^{6} \mathrm{~kg} \mathrm{~m}^{2} \mathrm{~s}^{-1}$. The difference between $v_{p t o, I G}$ and $v_{p t o, G F}$ is related to the behaviour of the exciting torque. Inspection of the different relations between radiation damping and exciting torque (Renzi and Dias [11]-Mei et al. [25]) shows that when $\omega$ is far from resonance the larger the exciting torque the larger the optimal PTO coefficient. In the present case the value $\omega=0.9 \mathrm{rad} \mathrm{s}^{-1}$ is very close to the peaks of the exciting torque for the gate farm (see Fig. 7), while is distant from the peak of the exciting torque for a single isolated gate (see Fig. 2). As a consequence, $v_{p t o, G F}$ is larger than $v_{p t o, I G}$. Hereafter, both $v_{p t o, G F}$ and $v_{p t o, I G}$ are fixed.
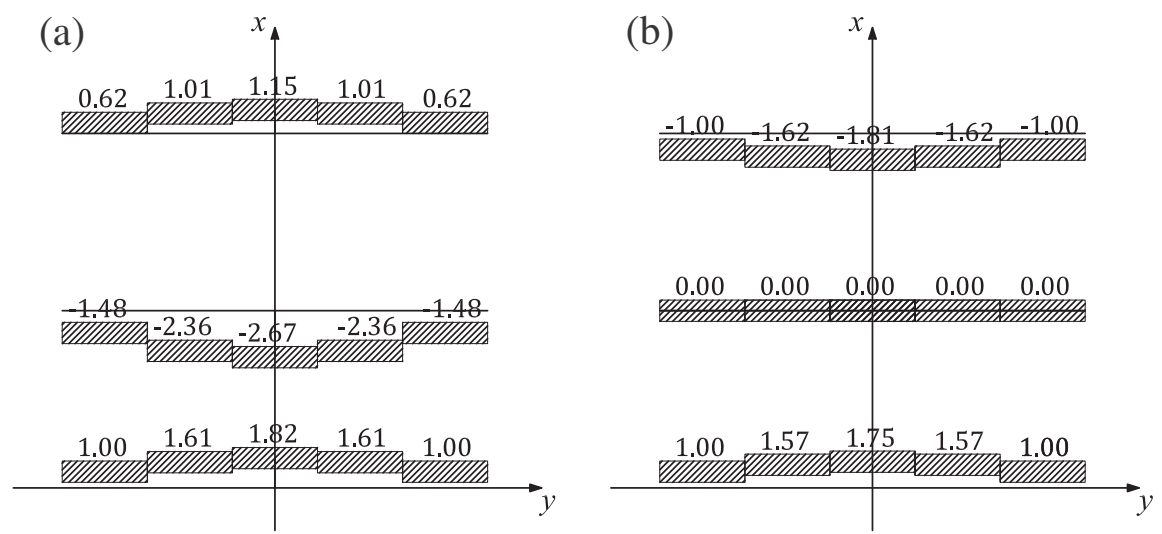

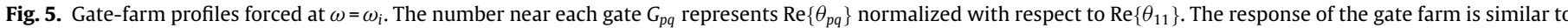
the modal form of the mode $N\left(\omega_{i}\right)$. (a) Response for $\omega=\omega_{1}$. (b) Response for $\omega=\omega_{2}$. 


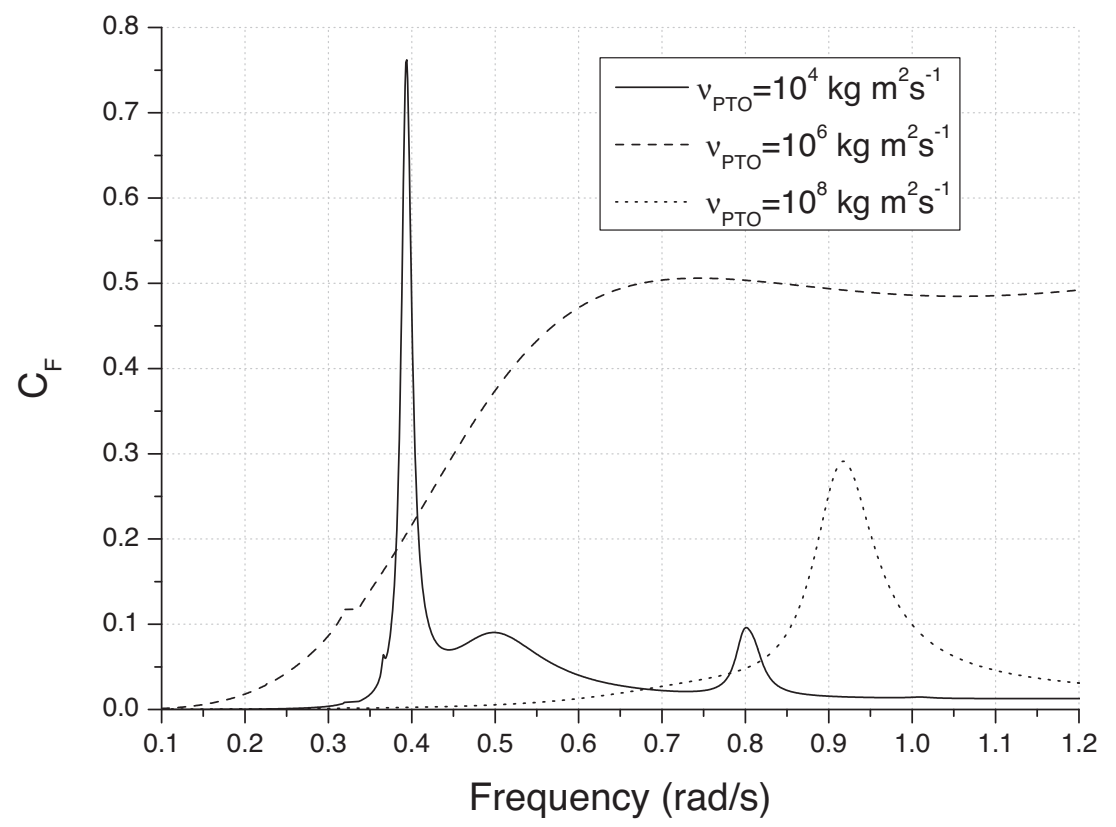

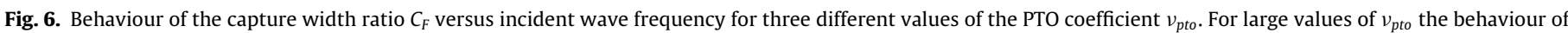
$C_{F}$ is dominated by the exciting torque due to diffracted waves. Differently, for small values of $v_{p t o}$ the behaviour of $C_{F}$ is dominated by the resonance effects.

Now define the capture width ratio of the gate farm $C_{G F}$ and the capture width ratio of $(P \times Q)$ isolated gates $C_{I G}$ as

$$
C_{G F}=\frac{P_{G F}}{\frac{1}{2} \rho g s A^{2} C_{g} a}, \quad C_{I G}=\frac{P_{I G}}{\frac{1}{2} \rho g A^{2} C_{g} a},
$$

where $P_{G F}$ and $P_{I G}$ represent respectively the averaged power generated by the gate farm and by the single isolated flap gate. Fig. 8 shows the capture width ratio curves of both systems. The gate farm captures significantly more energy than a system of isolated gates. Also the bandwidth of the gate farm curve is larger than the other. Note that $C_{G F}$ behaves as the exciting torque magnitude shown in Fig. 7 , hence the performance is dominated by diffracted waves. In Renzi et al. [15] have been obtained similar results.

Now consider the amplitude of the angular displacements $\theta_{33}$ of the gate $G_{33}$ and the amplitude of the angular displacements $\theta_{I G}$ of the isolated gate shown in Fig. 9. The maximum value for $\left|\theta_{33}\right|$ is $\sim 0.2 \mathrm{rad}$, hence the influence of the PTO coefficient decreases significantly the unrealistic amplitudes of the gates without PTO damping (see Fig. 2 for the gate farm). This fact justifies the hypothesis of small-amplitude oscillations and the applicability of the linear theory.

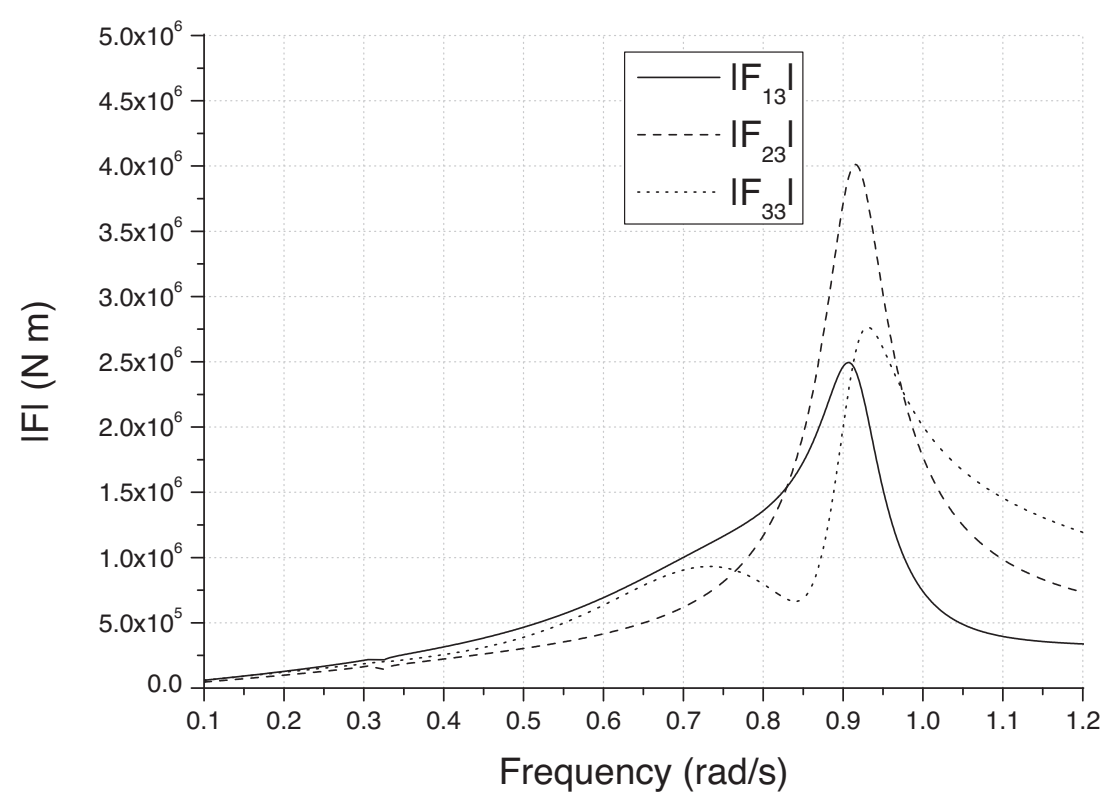

Fig. 7. Magnitude of the exciting torque $\left|F_{p 3}\right|$ on each flap gate $G_{p 3}$ versus incident wave frequency. 


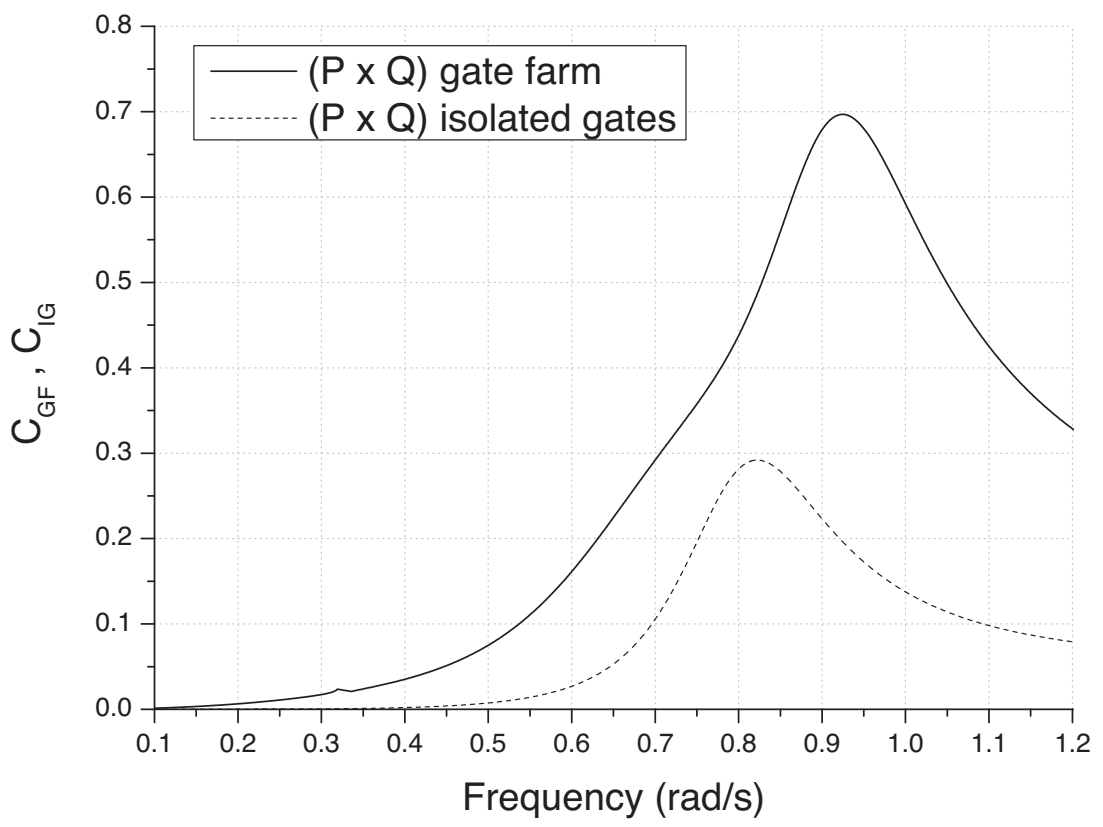

Fig. 8. Capture width ratio of the $(P \times Q)$ gate farm $C_{G F}$ and capture width ratio of $(P \times Q)$ isolated gates $C_{I G}$ versus incident wave frequency.

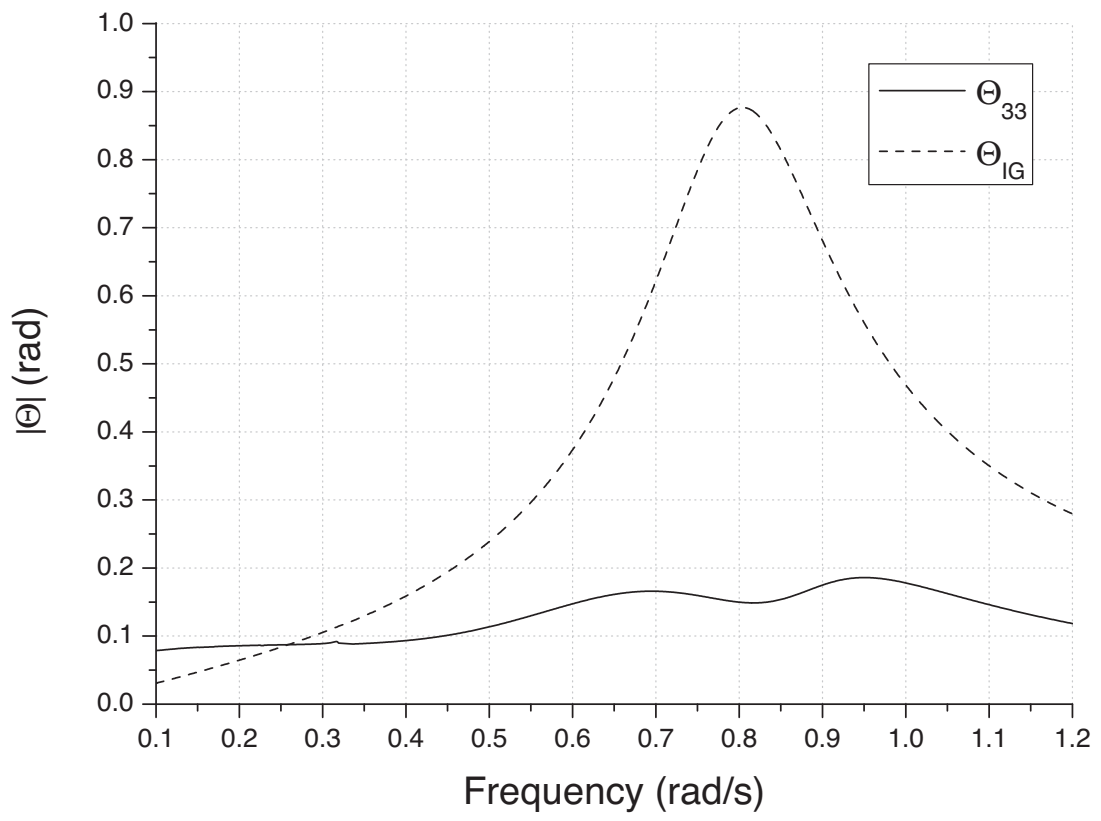

Fig. 9. Gate $G_{33}$ and isolated gate amplitude response versus incident wave frequency.

\section{Conclusions}

A semi-analytical model has been developed in order to solve the dynamic behaviour of the $P \times Q$ gate farm when excited by planar incident waves. By means of the Green theorem, a system of hypersingular integral equations for the radiation and scattering potential on the wet surfaces of the gate farm is obtained. The system is solved in terms of Legendre polynomials of integer order. Then the expressions of the added inertia, the radiation damping and the exciting torque are derived. The theory takes into account the thickness of each gate without resorting to the "thin-gate" hypothesis.

A parametric analysis of one gate in the open sea reveals the effect of the gate thickness on the eigenfrequency and on the gate response to incident waves. We have shown that the larger the thickness the larger the added inertia and the lower the eigenfrequency. Moreover, the radiation damping increases as the thickness increases, while the exciting torque shows negligible variations.

The solution of the eigenvalue condition for the $P \times Q$ gate farm, gives $P \times(Q-1)$ out-of-phase natural modes similar in shape to those of the $P \times Q$ gate farm in a channel of Sammarco et al. [6]. The system response is then evaluated for a wide range of incident wave frequencies. Numerical results show that the resonant peaks are close to the natural frequencies of the system. In particular, the narrow resonant peaks indicate that the radiation damping is small, hence synchronous excitation of the natural modes is significant. An asymptotic expression of 
the radiation potential is obtained in order to apply the Haskind-Hanaoka relation to the gate farm. The $(P \times Q)$ gate farm and a system of $(P \times Q)$ isolated gates are compared in terms of energy production. The results show that the gate farm capture more energy than a system of isolated gates.

The amplitude response at the resonance frequencies is large and non-realistic, hence the hypothesis of small-amplitude oscillation at the basis of this linear theory, is not satisfied. However, the amplitude response is significantly reduced when the gates are coupled with a PTO device at the hinge. Also fluid viscosity and vortex shedding should be considered in order to better evaluate dissipation effects (see Wei et al. [27]). For this reason, the development of a non-linear theory is necessary. This will also allow the evaluation of the gate response when the natural modes are excited sub-harmonically by incident waves.

\section{Acknowledgements}

The work of Frédéric Dias is supported by Science Foundation Ireland (SFI) under the research project "High-end computational modelling for wave energy systems" (Grant number SFI/10/IN.1/12996) in collaboration with Marine Renewable Energy Ireland (MaREI), the SFI Centre for Marine Renewable Energy Research - (12/RC/2302).

\section{Appendix}

Solution of the radiation and scattering potentials

For shorthand notations define the following integrals as follows:

$$
\begin{aligned}
& \left\{\begin{array}{l}
\mathcal{W}_{n p^{*} p q}^{R \pm} \\
\mathcal{W}_{n p^{*}}^{S \pm}
\end{array}\right\}=\left.\mp \int_{-\frac{w}{2}}^{\frac{w}{2}}\left\{\begin{array}{l}
\varphi_{n, p q}^{R}(\xi, \eta) \\
\varphi_{n}^{S}(\xi, \eta)
\end{array}\right\} \frac{\partial G_{n}}{\partial \xi}\right|_{\xi=\xi_{p^{*}}^{ \pm}} d \eta \\
& \left\{\begin{array}{l}
\mathcal{B}_{n p^{*}, p q}^{R \pm} \\
\mathcal{B}_{n p^{*}}^{S \pm}
\end{array}\right\}=\left.\mp \int_{\xi_{p^{*}}^{-}}^{\xi_{p^{*}}^{+}}\left\{\begin{array}{l}
\varphi_{n, p q}^{R}(\xi, \eta) \\
\varphi_{n}^{S}(\xi, \eta)
\end{array}\right\} \frac{\partial G_{n}}{\partial \eta}\right|_{\eta= \pm \frac{w}{2}} d \xi \\
& \mathcal{W}_{n, p q}^{R}=\mathrm{i} \omega \theta_{p q} f_{n} \int_{\eta_{q}}^{\eta_{q+1}}\left[\left.G_{n}\right|_{\xi=\xi_{p}^{-}}-\left.G_{n}\right|_{\xi=\xi_{p}^{+}}\right] d \eta, \\
& \mathcal{W}_{n p^{*}}^{S}=A d_{n} \int_{-\frac{w}{2}}^{\frac{w}{2}}\left[\left.e^{-\mathrm{i} k_{n} \xi} G_{n}\right|_{\xi=\xi_{p^{*}}}-\left.e^{-i k_{n} \xi} G_{n}\right|_{\xi=\xi_{p^{*}}}\right] d \eta,
\end{aligned}
$$

Imposing the boundary conditions (22a)-(22e) to the radiation and scattering potentials (32)-(33) yields:

$$
\begin{aligned}
& \frac{\partial \varphi_{n, p q}^{R}}{\partial x}=2 \frac{\partial}{\partial x}\left[\sum_{p^{*}=1}^{P}\left\{\mathcal{W}_{n p^{*}, p q}^{R+}+\mathcal{W}_{n p^{*}, p q}^{R-}+\mathcal{B}_{n p^{*}, p q}^{R+}+\mathcal{B}_{n p^{*}, p q}^{R-}\right\}+\mathcal{W}_{n, p q}^{R}\right]= \\
& \begin{cases}-\mathrm{i} \omega \theta_{p q} f_{n}, & \text { on } S_{p q}, \\
0, & \text { onS } \tilde{\mathrm{p}} \tilde{\mathrm{q}}, \tilde{\mathrm{p}} \neq \mathrm{p} \vee \tilde{\mathrm{q}} \neq \mathrm{q}, \quad(\mathrm{A} .5 \mathrm{~b})\end{cases} \\
& \frac{\partial \varphi_{n, p q}^{R}}{\partial y}=\frac{\partial}{\partial y}\left[\sum_{p^{*}=1}^{P}\left\{\mathcal{W}_{n p^{*}, p q}^{R+}+\mathcal{W}_{n p^{*}, p q}^{R-}+\mathcal{B}_{n p^{*}, p q}^{R+}+\mathcal{B}_{n p^{*}, p q}^{R-}\right\}+\mathcal{W}_{n, p q}^{R}\right]= \\
& =0, \quad \text { on } S_{\tilde{p}}, \quad \\
& \frac{\partial \varphi_{n}^{S}}{\partial x}=2 \frac{\partial}{\partial x}\left[\sum_{p^{*}=1}^{P}\left\{\mathcal{W}_{n p^{*}}^{+S}+\mathcal{W}_{n p^{*}}^{S-}+\mathcal{B}_{n p^{*}}^{+S}+\mathcal{B}_{n p^{*}}^{+S}+\mathcal{W}_{n p^{*}}^{S}\right\}\right]= \\
& =A d_{n} e^{-\mathrm{i} k_{n} x_{p}^{ \pm}}, \quad \text { on } S_{\tilde{p} \tilde{q},}, \\
& \frac{\partial \varphi_{n}^{S}}{\partial y}=\frac{\partial}{\partial y}\left[\sum_{p^{*}=1}^{P}\left\{\mathcal{W}_{n p^{*}}^{S}+\mathcal{W}_{n p^{*}}^{S-}+\mathcal{B}_{n p^{*}}^{+S}+\mathcal{B}_{n p^{*}}^{+S}+\mathcal{W}_{n p^{*}}^{S}\right\}\right]= \\
& =0, \quad \text { on } S_{\tilde{p}}, \\
& \tilde{p}=1, \ldots, P, \tilde{q}=1, \ldots, Q .
\end{aligned}
$$


Expressions (A.5a)-(A.6b) form two systems of $4 \times P$ integro-differential equations whose unknowns are respectively $\varphi_{n, p q}^{R}$ and $\varphi_{n}^{S}$ evaluated on the boundary of the gate farm. Consider the case where the index of the summation $p^{*}$ is equal to $\tilde{p}$. The integrals inside (A.5a)-(A.6b), given by

$$
\frac{\partial}{\partial x}\left\{\begin{array}{l}
\mathcal{W}_{n \tilde{p}, p q}^{R \pm} \\
\mathcal{W}_{n \tilde{p}}^{S \pm}
\end{array}\right\} \quad, \quad \frac{\partial}{\partial y}\left\{\begin{array}{l}
\mathcal{B}_{n \tilde{p}, p q}^{R \pm} \\
\mathcal{B}_{n \tilde{p}}^{S \pm}
\end{array}\right\}
$$

are hypersingular when $\eta= \pm y$ and $\xi= \pm x$. In this case, the inversion between the outer derivative and the integral sign is possible by means of the Hadamard finite-part integral $\mathrm{H} \int$.

Recalling the expression of the Hankel function $H_{1}^{(1)}$ [26]

$$
H_{1}^{(1)}(\alpha)=-\frac{2 \mathrm{i}}{\alpha \pi}+R_{n}(\alpha)
$$

where

$$
R_{n}(\alpha)=J_{1}(\alpha)+\frac{\mathrm{i}}{\pi}\left\{2 J_{1}(\alpha)\left(\frac{\ln \alpha}{2}+\gamma\right)-\frac{\alpha}{2}-\sum_{k=2}^{\infty}(-1)^{k+1} \frac{(\alpha / 2)^{2 k-1}}{k !(k-1) !}\left(\frac{1}{k}+2 \sum_{m=1}^{k-1} \frac{1}{m}\right)\right\},
$$

with $J_{1}(\alpha)$ the Bessel function of the first kind and order 1 and $\gamma$ the Euler-Mascheroni constant, the integrals in (A.7) can be rewritten as:

$$
\begin{aligned}
& \frac{\partial}{\partial x}\left\{\begin{array}{l}
\mathcal{W}_{n \tilde{p}, p q}^{R \pm} \\
\mathcal{W}_{n \tilde{p}}^{S \pm}
\end{array}\right\}= \pm \frac{1}{2 \pi} \mathrm{H} \int_{-\frac{w}{2}}^{\frac{w}{2}}\left\{\begin{array}{l}
\varphi_{n, p q}^{R} \\
\varphi_{n}^{S}
\end{array}\right\}_{\xi=\xi_{\tilde{p}}^{ \pm}}(y-\eta)^{-2} d \eta \mp\left\{\begin{array}{l}
\mathcal{L}^{R \pm}\left(\varphi_{n, p q}^{R}\right) \\
\mathcal{L}^{S \pm}\left(\varphi_{n}^{S}\right)
\end{array}\right\} \quad \text { on } S_{\tilde{p} \tilde{q}}, \\
& \frac{\partial}{\partial y}\left\{\begin{array}{l}
\mathcal{B}_{n \tilde{p}, p q}^{R \pm} \\
\mathcal{B}_{n \tilde{p}}^{S \pm}
\end{array}\right\} \pm \frac{1}{2 \pi} \mathrm{H} \int_{\xi_{\tilde{p}}^{-}}^{\xi_{\tilde{p}}^{+}}\left\{\begin{array}{l}
\varphi_{n, p q}^{R} \\
\varphi_{n}^{S}
\end{array}\right\}_{\eta= \pm \frac{w}{2}}(x-\xi)^{-2} d \xi \mp\left\{\begin{array}{l}
\mathcal{T}^{R \pm}\left(\varphi_{n, p q}^{R}\right) \\
\mathcal{T}^{S \pm}\left(\varphi_{n}^{S}\right)
\end{array}\right\} \quad \text { on } S_{\tilde{p}},
\end{aligned}
$$

where

$$
\begin{gathered}
\left\{\begin{array}{l}
\mathcal{L}^{R \pm}\left(\varphi_{n, p q}^{R}\right) \\
\mathcal{L}^{S \pm}\left(\varphi_{n}^{S}\right)
\end{array}\right\}=\int_{-\frac{w}{2}}^{\frac{w}{2}}\left\{\begin{array}{l}
\varphi_{n, p q}^{R} \\
\varphi_{n}^{S}
\end{array}\right\}_{\xi=\xi_{\tilde{p}}^{ \pm}} \frac{k_{n} \mathrm{i} R_{n}\left(k_{n}|y-\eta|\right)}{4|y-\eta|} d \eta \\
\left\{\begin{array}{l}
\mathcal{T}^{R \pm}\left(\varphi_{n, p q}^{R}\right) \\
\mathcal{T}^{\mathcal{I}}\left(\varphi_{n}^{S}\right)
\end{array}\right\}=\int_{\xi_{\tilde{p}}^{-}}^{\xi_{\tilde{p}}^{+}}\left\{\begin{array}{l}
\varphi_{n, p q}^{R} \\
\varphi_{n}^{S}
\end{array}\right\}_{\eta= \pm \frac{w}{2}} \frac{k_{n} \mathrm{i} R_{n}\left(k_{n}|x-\xi|\right)}{4|x-\xi|} d \xi .
\end{gathered}
$$

Note that when $|y-\eta| \rightarrow 0$ and $|x-\xi| \rightarrow 0, R_{n}\left(k_{n}|y-\eta|\right) \simeq|y-\eta| \ln |y-\eta|$ and $R_{n}\left(k_{n}|x-\xi|\right) \simeq|x-\xi| \ln |x-\xi|$, hence, both $\mathcal{L}^{ \pm,(R, S)}$ and $\mathcal{T}^{ \pm,(R, S)}$ are not singular. In order to simplify notations, rewrite (A.10)-(A.11) as:

$$
\frac{\partial}{\partial x}\left\{\begin{array}{l}
\mathcal{W}_{n \tilde{p}, p q}^{R \pm} \\
\mathcal{W}_{n \tilde{p}}^{S \pm}
\end{array}\right\}=\left\{\begin{array}{c}
\mathcal{I}_{n \tilde{p}, p q}^{R \pm} \\
\mathcal{I}_{n \tilde{p}}^{S \pm}
\end{array}\right\}, \quad \frac{\partial}{\partial y}\left\{\begin{array}{l}
\mathcal{B}_{n \tilde{p}, p q}^{R \pm} \\
\mathcal{B}_{n \tilde{p}}^{S \pm}
\end{array}\right\}=\left\{\begin{array}{l}
\mathcal{H}_{n \tilde{p}, p q}^{R \pm} \\
\mathcal{H}_{n \tilde{p}}^{S \pm}
\end{array}\right\}
$$

define $x_{p}$ and $\xi_{p}$ as follows:

$$
x_{p}=x-(p-1) L, \quad \xi_{p}=\xi-(p-1) L,
$$

and introduce the dimensionless variables denoted by primes:

$$
\eta^{\prime}=\frac{2 \eta}{w}, \quad y^{\prime}=\frac{2 y}{w}, \quad \xi_{p}^{\prime}=\frac{\xi_{p}}{b}, \quad x_{p}^{\prime}=\frac{x_{p}}{b} .
$$

The radiation and scattering potentials on the boundary of each array $\bar{p}=1, \ldots, P$, can be expressed in terms of the new functions $f$ and $g$ each defined in the interval $[-1,1]$ :

$$
\begin{aligned}
& \left\{\begin{array}{l}
\varphi_{n, p q}^{R}\left(\xi=\xi_{\bar{p}}^{ \pm}, \eta\right) \\
\varphi_{n}^{S}\left(\xi=\xi_{\bar{p}}^{ \pm}, \eta\right)
\end{array}\right\}=\left\{\begin{array}{l}
\varphi_{n, p q}^{R}\left(\xi_{\bar{p}}= \pm b, \eta\right) \\
\varphi_{n}^{S}\left(\xi_{\bar{p}}= \pm b, \eta\right)
\end{array}\right\}=\left\{\begin{array}{l}
f_{n \bar{p}, p q}^{R \pm}\left(\eta^{\prime}\right) \\
f_{n \bar{p}}^{S \pm}\left(\eta^{\prime}\right)
\end{array}\right\}, \\
& \left\{\begin{array}{l}
\varphi_{n, p q}^{R}\left(\xi, \eta= \pm \frac{w}{2}\right) \\
\varphi_{n}^{S}\left(\xi, \eta= \pm \frac{w}{2}\right)
\end{array}\right\}=\left\{\begin{array}{l}
g_{n \bar{p}, p q}^{R \pm}\left(\xi_{\bar{p}}^{\prime}\right) \\
g_{n \bar{p}}^{S \pm}\left(\xi_{\bar{p}}^{\prime}\right)
\end{array}\right\} .
\end{aligned}
$$

According to (A.15), (A.16), (A.17) and (A.18), expressions (A.1) and (A.2) become:

$$
\left\{\begin{array}{l}
\mathcal{W}_{n p^{*}, p q}^{R \pm} \\
\mathcal{W}_{n p^{*}}^{S \pm}
\end{array}\right\}=\left.\mp \frac{w}{2 b} \int_{-1}^{1}\left\{\begin{array}{l}
f_{n p^{*}, p q}^{R \pm} \\
f_{n p^{*}}^{S \pm}
\end{array}\right\} \frac{\partial G_{n}}{\partial \xi_{p^{*}}^{\prime}}\right|_{\xi_{p^{*}}^{\prime}= \pm 1} d \eta^{\prime}
$$




$$
\left\{\begin{array}{l}
\mathcal{B}_{n p^{*}, p q}^{R \pm} \\
\mathcal{B}_{q p^{*}}^{S \pm}
\end{array}\right\}=\left.\mp \frac{2 b}{w} \int_{-1}^{1}\left\{\begin{array}{l}
g_{n p^{*}, p q}^{R \pm} \\
g_{n p^{*}}^{S \pm}
\end{array}\right\} \frac{\partial G_{n}}{\partial \eta^{\prime}}\right|_{\eta^{\prime}= \pm 1} d \xi_{p^{*}}^{\prime},
$$

while the expressions (A.10)-(A.11) including the singular part can be written as:

$$
\begin{aligned}
& \left\{\begin{array}{l}
\mathcal{I}_{n \tilde{p}, p q}^{R \pm} \\
\mathcal{I}_{n \tilde{p}}^{S \pm}
\end{array}\right\}= \pm \frac{1}{w \pi} \mathrm{H} \int_{-1}^{1}\left\{\begin{array}{l}
f_{n \tilde{p}, p q}^{R \pm} \\
f_{n \tilde{p}}^{S \pm}
\end{array}\right\}\left(y^{\prime}-\eta^{\prime}\right)^{-2} d \eta^{\prime} \mp\left\{\begin{array}{l}
\mathcal{L}^{R \pm}\left(f_{n \tilde{p}, p q}^{R \pm}\right) \\
\mathcal{L}^{S \pm}\left(f_{n \tilde{p}}^{S \pm}\right)
\end{array}\right\}, \\
& \left\{\begin{array}{l}
\mathcal{H}_{n \tilde{p}, p q}^{R \pm} \\
\mathcal{H}_{n \tilde{p}}^{S \pm}
\end{array}\right\}= \pm \frac{1}{2 \pi b} \mathrm{H} \int_{-1}^{1}\left\{\begin{array}{l}
g_{n \tilde{p}, p q}^{R \pm} \\
g_{n \tilde{p}}^{S \pm}
\end{array}\right\}\left(x_{\tilde{p}}^{\prime}-\xi_{\tilde{p}}^{\prime}\right)^{-2} d \xi_{\tilde{p}}^{\prime} \mp\left\{\begin{array}{l}
\mathcal{T}^{R \pm}\left(g_{n \tilde{p}, p q}^{R \pm}\right) \\
\mathcal{T}^{S \pm}\left(g_{n \tilde{p}}^{R \pm}\right)
\end{array}\right\} .
\end{aligned}
$$

In order to solve the hypersingular integrals, let us seek solutions of the type:

$$
\begin{aligned}
& \left\{\begin{array}{l}
f_{n \bar{p}, p q}^{R \pm} \\
f_{n \bar{p}}^{S \pm}
\end{array}\right\}=\sum_{m=0}^{M}\left\{\begin{array}{l}
\alpha_{n m \bar{p}, p q}^{R \pm} P_{m} \theta_{p q} \\
\alpha_{n m \bar{p}}^{S \pm} P_{m}
\end{array}\right\}, \\
& \left\{\begin{array}{l}
g_{n \bar{p}, p q}^{R \pm} \\
g_{n \bar{p}}^{S \pm}
\end{array}\right\}=\sum_{m=0}^{M}\left\{\begin{array}{l}
\beta_{n m \bar{p}, p q}^{R \pm} P_{m} \theta_{p q} \\
\beta_{n m \bar{p}}^{S \pm} P_{m}
\end{array}\right\},
\end{aligned}
$$

where $\alpha_{n m \bar{p}, p q}^{R \pm}, \alpha_{n m \bar{p}}^{S \pm}, \beta_{n m \bar{p}, p q}^{R \pm}$ and $\beta_{n m \bar{p}}^{S_{ \pm}}$are unknown complex constants, $P_{m}$ are the Legendre polynomials of order $m$ with $m \in \mathbb{N}$ and $M$ is a finite integer. The proposed expansion is motivated by the works of Renzi and Dias [9] and Parsons and Martin [20] who have used Chebyshev polynomials to represent scattering and radiation potential on the thin-gate" surface. This expansion respects the behaviour of the jump in potential $\Delta \varphi$ near the end-points of the flap, i.e. $\Delta \varphi \rightarrow 0$ (Renzi and Dias [9]). However, differently from the case of the thin gate, the behaviour at the corners of the gate farm (i.e. the counterpart of the "end-points") is unknown, hence we cannot use Chebyshev expansion.

Legendre polynomials are advantageous in that, the related hypersingular integral, interpreted as a finite-part integral, can be evaluated in the closed form. Another feature of using Legendre polynomials is that the values of the potential can be determined throughout a low computation effort; see for example Kolm and Rokhlin [28], Yang [29] and Carley [30], who also employ Legendre polynomials.

By definition of Hadamard integral, the hypersingular integrals inside expressions (A.21)-(A.22) then become:

$$
\begin{aligned}
& \mathrm{H} \int_{-1}^{1}\left\{\begin{array}{l}
f_{n \tilde{p}, p q}^{R \pm} \\
f_{n \tilde{p}}^{S \pm}
\end{array}\right\}\left(y^{\prime}-\eta^{\prime}\right)^{-2} d \eta^{\prime}=\frac{d}{d y^{\prime}} \mathrm{P} \int_{-1}^{1}\left\{\begin{array}{c}
f_{n \tilde{p}, p q}^{R \pm} \\
f_{n \tilde{p}}^{S \pm}
\end{array}\right\}\left(y^{\prime}-\eta^{\prime}\right)^{-1} d \eta^{\prime}, \\
& \mathrm{H} \int_{-1}^{1}\left\{\begin{array}{l}
g_{n \tilde{p}, p q}^{R \pm} \\
g_{n \tilde{p}}^{S \pm}
\end{array}\right\}\left(x_{\tilde{p}}^{\prime}-\xi_{\tilde{p}}^{\prime}\right)^{-2} d \xi_{\tilde{p}}^{\prime}=\frac{d}{d x^{\prime}} \mathrm{P} \int_{-1}^{1}\left\{\begin{array}{l}
g_{n \tilde{p}, p q}^{R \pm} \\
g_{n \tilde{p}}^{S \pm}
\end{array}\right\}\left(x_{\tilde{p}}^{\prime}-\xi_{\tilde{p}}^{\prime}\right)^{-1} d \xi_{\tilde{p}}^{\prime},
\end{aligned}
$$

where P $\int$ is the Cauchy principal-value integral. Now consider the integral relation (Kaya and Erdogan [31] expression (27)):

$$
\mathrm{P} \int_{-1}^{1} \frac{P_{m}(\psi)}{\psi-\tau} d \psi=-2 Q_{m}(\tau), \quad-1<\tau<1
$$

where $Q_{m}$ are the Legendre functions of the second kind and order $m$. Substitution of the series expansions (A.23)-(A.24) in the (A.25)-(A.26) yields:

$$
\begin{aligned}
& \frac{d}{d y^{\prime}} \mathrm{P} \int_{-1}^{1}\left\{f_{n \tilde{p}, p q}^{R \pm} f_{n \tilde{p}}^{S \pm}\right\}\left(y^{\prime}-\eta^{\prime}\right)^{-1} d \eta^{\prime}=\sum_{m=0}^{M}\left\{\alpha_{n m \tilde{p}, p q}^{R \pm} \theta_{p q} \alpha_{n m \tilde{p}}^{S \pm}\right\}\left[-2(m+1) \frac{y^{\prime} Q_{m}\left(y^{\prime}\right)-Q_{m+1}\left(y^{\prime}\right)}{1-y^{\prime 2}}\right], \\
& \frac{d}{d x^{\prime}} \mathrm{P} \int_{-1}^{1}\left\{g_{n \tilde{p}, p q}^{R \pm} g_{n \tilde{p}}^{S \pm}\right\}\left(x_{\tilde{p}}^{\prime}-\xi_{\tilde{p}}^{\prime}\right)^{-1} d \xi_{\tilde{p}}^{\prime}=\sum_{m=0}^{M}\left\{\beta_{n m \tilde{p}, p q}^{R \pm} \theta_{p q} \beta_{n m \tilde{p}}^{S \pm}\right\}\left[-2(m+1) \frac{x^{\prime} Q_{m}\left(x^{\prime}\right)-Q_{m+1}\left(x^{\prime}\right)}{1-x^{\prime 2}}\right] .
\end{aligned}
$$

Finally the hypersingular integrals are solved in terms of Legendre polynomials, hence (A.21) and (A.22) become:

$$
\begin{gathered}
\left\{\begin{array}{l}
\mathcal{I}_{n \tilde{p}, p q}^{R \pm} \\
\mathcal{I}_{n \tilde{p}}^{S \pm}
\end{array}\right\}=\sum_{m=0}^{M}\left\{\begin{array}{l}
\alpha_{n m \tilde{p}, p q}^{R \pm} \theta_{p q} \\
\alpha_{n m \tilde{p}}^{S \pm}
\end{array}\right\}\left\{\begin{array}{l}
\widetilde{\mathcal{I}}_{m}^{R \pm} \\
\widetilde{\mathcal{I}}_{m}^{S \pm}
\end{array}\right\}, \\
\left\{\begin{array}{l}
\mathcal{H}_{n \tilde{p}, p q}^{R \pm} \\
\mathcal{H}_{n \tilde{p}}^{S \pm}
\end{array}\right\}=\sum_{m=0}^{M}\left\{\begin{array}{l}
\beta_{n m \tilde{p}, p q}^{R \pm} \theta_{p q} \\
\beta_{n m \tilde{p}}^{S \pm}
\end{array}\right\}\left\{\begin{array}{l}
\widetilde{\mathcal{H}}_{m}^{R \pm} \\
\widetilde{\mathcal{H}}_{m}^{S \pm}
\end{array}\right\},
\end{gathered}
$$


where:

$$
\begin{aligned}
& \left\{\begin{array}{c}
\widetilde{\mathcal{I}}_{m}^{R \pm} \\
\widetilde{\mathcal{I}}_{m}^{S_{ \pm}}
\end{array}\right\}=\mp \frac{2}{w \pi}\left[(m+1) \frac{y^{\prime} Q_{m}\left(y^{\prime}\right)-Q_{m+1}\left(y^{\prime}\right)}{1-y^{2}}\right] \mp\left\{\begin{array}{l}
\mathcal{L}^{R \pm}\left(P_{m}\right) \\
\mathcal{L}^{S \pm}\left(P_{m}\right)
\end{array}\right\}, \\
& \left\{\begin{array}{c}
\widetilde{\mathcal{H}}_{m}^{R \pm} \\
\widetilde{\mathcal{H}}_{m}^{S \pm}
\end{array}\right\}=\mp \frac{1}{b \pi}\left[(m+1) \frac{x^{\prime} Q_{m}\left(x^{\prime}\right)-Q_{m+1}\left(x^{\prime}\right)}{1-x^{\prime 2}}\right] \mp\left\{\begin{array}{l}
\mathcal{T}^{R \pm}\left(P_{m}\right) \\
\mathcal{T}^{S \pm}\left(P_{m}\right)
\end{array}\right\} .
\end{aligned}
$$

The expressions (A.19) and (A.20) which include the functions $f$ and $g$, after substitution of (A.23)-(A.24) are given by:

$$
\begin{aligned}
& \left\{\mathcal{W}_{n p^{*}, p q}^{R \pm} \mathcal{W}_{n p^{*}}^{S \pm}\right\}=\left.\mp \frac{w}{2 b} \sum_{m=0}^{M}\left\{\alpha_{n m p^{*}, p q}^{R \pm} \theta_{p q} \alpha_{n m p^{*}}^{S \pm}\right\} \int_{-1}^{1} P_{m}\left(\eta^{\prime}\right) \frac{\partial G_{n}}{\partial \xi_{p^{*}}^{\prime}}\right|_{\xi_{p^{*}}^{\prime}= \pm 1} d \eta^{\prime}==\frac{w}{2 b} \sum_{m=0}^{M}\left\{\alpha_{n m p^{*}, p q}^{R \pm} \theta_{p q} \alpha_{n m p^{*}}^{S \pm}\right\}\left\{\widetilde{\mathcal{W}}_{m p^{*}}^{R \pm} \widetilde{\mathcal{W}}_{m p^{*}}^{S \pm}\right\}, \\
& \left\{\mathcal{B}_{n p^{*}, p q}^{R \pm} \mathcal{B}_{n p^{*}}^{S \pm}\right\}=\left.\mp \frac{2 b}{w} \sum_{m=0}^{M}\left\{\beta_{n m p^{*}, p q}^{R \pm} \theta_{p q} \beta_{n m p^{*}}^{S \pm}\right\} \int_{-1}^{1} P_{m}\left(\xi_{p^{*}}^{\prime}\right) \frac{\partial G_{n}}{\partial \eta^{\prime}}\right|_{\eta^{\prime}= \pm 1} d \xi_{p^{*}}^{\prime}==\frac{2 b}{w} \sum_{m=0}^{M}\left\{\beta_{n m p^{*}, p q}^{R \pm} \theta_{p q} \beta_{n m p^{*}}^{S \pm}\right\}\left\{\widetilde{\mathcal{B}}_{m p^{*}}^{R \pm} \widetilde{\mathcal{B}}_{m p^{*}}^{S \pm}\right\} .
\end{aligned}
$$

Define the normalized boundaries $S_{p q}^{\prime}$ and $S_{p}^{\prime}$ as follows:

$$
\begin{aligned}
& S_{p q}^{\prime}=\left\{x_{p}^{\prime}= \pm 1, y \in\left[\frac{2 y_{q}}{w}, \frac{2 y_{q+1}}{w}\right]\right\}, \\
& S_{p}^{\prime}=\left\{x_{p}^{\prime} \in[-1,1], y^{\prime}= \pm 1\right\},
\end{aligned}
$$

the two system (A.5a)-(A.5c) and (A.6a)-(A.6b) can be rewritten as:

$$
\begin{aligned}
& \frac{\partial}{\partial x_{\tilde{p}}^{\prime}}\left\{\sum_{p^{*}=1}^{P} \sum_{m=0}^{M}\left\{\alpha_{n m p^{*}, p q}^{\mp, R} \theta_{p q} \widetilde{\mathcal{W}}_{m p^{*}}^{\mp, R}+\beta_{n m p^{*}, p q}^{R+} \theta_{p q} \widetilde{\mathcal{B}}_{m p^{*}}^{R+}+\beta_{n m p^{*}, p q}^{R-} \theta_{p q} \widetilde{\mathcal{B}}_{m p^{*}}^{R-}\right\}+\right. \\
& \left.\sum_{p^{*}=1}^{P} \sum_{m=0}^{M} \alpha_{n m p^{*}, p q}^{R \pm} \theta_{p q} \widetilde{\mathcal{W}}_{m p^{*}}^{R \pm}+\mathcal{W}_{n, p q}^{R}\right\}+\sum_{m=0}^{M} \alpha_{n m \tilde{p}, p q}^{R \pm} \theta_{p q} \widetilde{\mathcal{I}}_{m}^{R \pm}= \\
& p^{*} \neq \tilde{p} \\
& \left\{\begin{array}{ccc}
-\frac{\mathrm{i} \omega \theta_{p q} f_{n}}{2}, & \text { on } S_{p q}^{\prime}, & \text { (A.38a) } \\
0, & \text { on } S_{\tilde{p} \tilde{q}}^{\prime}, \tilde{p} \neq p \vee \tilde{q} \neq q, & \text { (A.38b) }
\end{array}\right. \\
& \frac{\partial}{\partial y^{\prime}}\left\{\sum_{p^{*}=1}^{P} \sum_{m=0}^{M}\left\{\beta_{n m p^{*}, p q}^{\mp, R} \theta_{p q} \widetilde{\mathcal{B}}_{m p^{*}}^{\mp, R}+\alpha_{n m p^{*}, p q}^{R+} \theta_{p q} \widetilde{\mathcal{W}}_{m p^{*}}^{R+}+\alpha_{n m p^{*}, p q}^{R-} \theta_{p q} \widetilde{\mathcal{W}}_{m p^{*}}^{R-}\right\}+\sum_{p^{*}=1}^{P} \sum_{m=0}^{M} \beta_{n m p^{*}, p q}^{R \pm} \theta_{p q} \widetilde{\mathcal{W}}_{m p^{*}}^{R \pm}+\mathcal{W}_{n, p q}^{R}\right\}+\sum_{m=0}^{M} \beta_{n m \tilde{p}, p q}^{R \pm} \theta_{p q} \widetilde{\mathcal{H}}_{m}^{R \pm}= \\
& =0, \quad \text { on } S_{\tilde{p}}^{\prime} \text {, } \\
& p^{*} \neq \tilde{p} \\
& \frac{\partial}{\partial x_{\tilde{p}}^{\prime}}\left\{\sum_{p^{*}=1}^{P} \sum_{m=0}^{M}\left\{\alpha_{n m p^{*}}^{\mp, S} \widetilde{\mathcal{W}}_{m p^{*}}^{\mp, S}+\beta_{n m p^{*}}^{+S} \widetilde{\mathcal{B}}_{m p^{*}}^{+S}+\beta_{n m p^{*}}^{S-} \widetilde{\mathcal{B}}_{m p^{*}}^{S_{-}}+\mathcal{W}_{n p^{*}}^{S}\right\}+\sum_{p^{*}=1}^{P} \sum_{m=0}^{M} \alpha_{n m p^{*}}^{S \pm} \widetilde{\mathcal{W}}_{m p^{*}}^{S_{ \pm}}\right\} \quad+\sum_{m=0}^{M} \alpha_{n m \tilde{p}}^{S_{ \pm}} \widetilde{\mathcal{I}}_{m}^{S_{ \pm}}= \\
& p^{*} \neq \tilde{p} \quad x_{\tilde{p}}^{\prime}= \pm 1 \\
& =\frac{A d_{n} e^{-i k_{n} x_{\tilde{p}}^{ \pm}}}{2}, \quad \text { on } S_{\tilde{p} \tilde{q}}^{\prime} \\
& \frac{\partial}{\partial y^{\prime}}\left\{\sum_{p^{*}=1}^{P} \sum_{m=0}^{M}\left\{\beta_{n m p^{*}}^{\mp, S} \widetilde{\mathcal{B}}_{m p^{*}}^{\mp, S}+\alpha_{n m p^{*}}^{+S} \widetilde{\mathcal{W}}_{m p^{*}}^{+S}+\alpha_{n m p^{*}}^{S-} \widetilde{\mathcal{W}}_{m p^{*}}^{S-}+\mathcal{W}_{n p^{*}}^{S}\right\}+\sum_{p^{*}=1}^{P} \sum_{m=0}^{M} \beta_{n m p^{*}}^{S_{ \pm}} \widetilde{\mathcal{B}}_{m p^{*}}^{S_{ \pm}}\right\}+\sum_{m=0}^{M} \beta_{n m p}^{S_{ \pm}} \widetilde{\mathcal{H}}_{m}^{S_{ \pm}^{ \pm}}= \\
& =0, \quad \text { on } S_{\tilde{p}}^{\prime} \text {, } \\
& p^{*} \neq \tilde{p}
\end{aligned}
$$


Expressions (A.38a)-(A.38c) and (A.39a)-(A.39b) define two systems of linear equations whose unknowns are respectively $\alpha_{n m p^{*}, p q}^{R \pm}$ and $\beta_{n m p^{*}, p q}^{R \pm}$ for the radiation problem, $\alpha_{n m p *}^{S \pm}$ and $\beta_{n m p^{*}}^{S \pm}$ for the scattering problem. Each system has $4 \times P \times M+1$ unknowns, hence $M+1$ evaluation points must be chosen for each side of the single array. A good choice for the collocation points $\left(x_{p, j}, y_{j}\right)$ is given by the roots of Chebyshev polynomials of the first kind (Parsons and Martin [20]-Kaya and Erdogan [31]) i.e.

$$
\begin{aligned}
& \left(x_{p, j}, y_{j}\right)=\left(b \cos \frac{(2 j+1) \pi}{2 M+2}-(p-1) L, \pm \frac{w}{2}\right), \\
& \left(x_{p, j}, y_{j}\right)=\left( \pm b-(p-1) L, \frac{w}{2} \cos \frac{(2 j+1) \pi}{2 M+2}\right), \\
& j=0,1, \ldots, M, p=1, \ldots, P .
\end{aligned}
$$

Systems (A.38a)-(A.38c) and (A.39a)-(A.39b) can be solved numerically for each modal order $n=0,1, \ldots$, therefore the radiation potential $\phi_{p q}^{R}$ and the scattering potential $\phi^{S}$ on the boundary of the $\tilde{p}$ th array, are given by:

$$
\begin{aligned}
& \left\{\begin{array}{l}
\phi_{p q}^{R}\left(x_{\tilde{p}}^{ \pm}, y, z\right) \\
\phi_{p q}^{R}\left(x, \pm \frac{w}{2}, z\right)
\end{array}\right\}=\sum_{n=0}^{\infty} \sum_{m=0}^{M} Z_{n}(z) \theta_{p q}\left\{\begin{array}{l}
P_{m}\left(y^{\prime}\right) \alpha_{n m \tilde{p}, p q}^{R \pm} \\
P_{m}\left(x_{\tilde{p}}^{\prime}\right) \beta_{n m \tilde{p}, p q}^{R \pm}
\end{array}\right\}, \\
& \left\{\begin{array}{l}
\phi^{S}\left(x_{\tilde{p}}^{ \pm}, y, z\right) \\
\phi^{S}\left(x, \pm \frac{w}{2}, z\right)
\end{array}\right\}=\sum_{m=0}^{M} Z_{0}(z)\left\{\begin{array}{l}
P_{m}\left(y^{\prime}\right) \alpha_{0 m \tilde{p}}^{S \pm} \\
P_{m}\left(x_{\tilde{p}}^{\prime}\right) \beta_{0 m \tilde{p}}^{S \pm}
\end{array}\right\},
\end{aligned}
$$

Note that the complex coefficients $\alpha_{n m \tilde{p}}^{S \pm}$ and $\beta_{n m \tilde{p}}^{S \pm}$ for $n=1,2, \ldots$, are equal to zero.

\section{References}

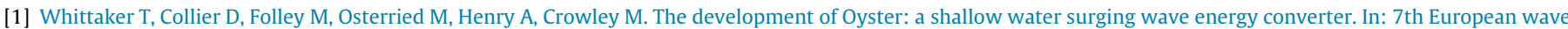
and tidal energy conference. 2007.

[2] Mei CC, Sammarco P, Chan ES, Procaccini C. Subharmonic resonance of proposed storm gates for Venice lagoon. Proc R Soc Lond A 1994;444:257-65.

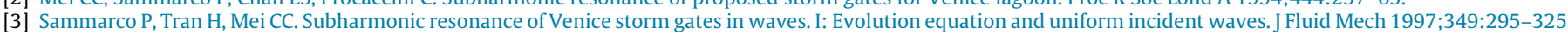

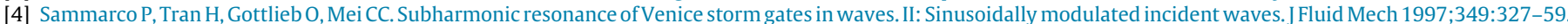

[5] Li G, Mei CC. Natural modes of mobile flood gates. Appl Ocean Res 2003;25:115-26.

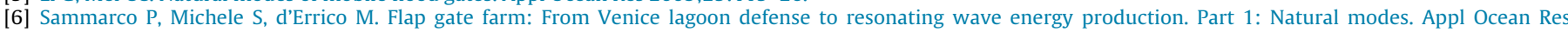
2013;43:206-13.

[7] Adamo A. Mei CC. Linear response of Venice storm gates to incident waves. Proc R Soc Lond A 2005;461:1711-34

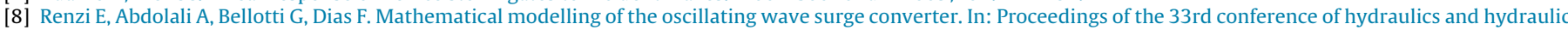
engineering. 2012.

[9] Renzi E, Dias F. Resonant behaviour of an oscillating wave energy converter in a channel. J Fluid Mech 2012;701:482-510.

[10] Renzi E, Dias F. Hydrodynamics of the oscillating wave surge converter in the open ocean. Eur J Mech B/Fluids 2013;41:1-10.

[11] Renzi E, Dias F. Relations for a periodic array of flap-type wave energy converters. Appl Ocean Res 2012;39:31-9.

[12] Renzi E, Dias F. Resonant scattering by a periodic array of plates with application to wave energy extraction. In: Proceedings of the IWWWFB27. 2012. p. 157-60.

[13] Renzi E, Dias F. Motion resonant modes of large articulated damped oscillators in waves. J Fluid Struct 2014:49:705-15.

[14] Renzi E, Abdolali A, Bellotti G, Dias F. Wave power absorption from a finite array of oscillating wave surge converters. Renew Energy 2014;63:55-68.

[15] Renzi E, Doherty K, Henry H, Dias F. How does Oyster work? The simple interpretation of Oyster mathematics. Eur J Mech B/Fluids 2014;47:124-31.

[16] Sarkar D, Renzi E, Dias F. Wave farm modelling of oscillating wave surge converters. Proc R Soc Lond A 2014;470:20140118

[17] Linton CM, McIver P. Mathematical techniques for wave/structure interactions. Chapman \& Hall/CRC; 2001.

[18] Achenbach JD, Li ZL. Reflection and transmission of scalar waves by a periodic array of screens. Wave Motion 1986;8:225-34.

[19] Martin PA, Rizzo FJ. On boundary integral equations for crack problems. Proc R Soc Lond A 1989;421:341-55.

[20] Parsons NF, Martin PA. Scattering of water waves by submerged plates using hypersingular integral equations. Appl Ocean Res 1992;14:313-21.

[21] Parsons NF, Martin PA. Scattering of water waves by submerged curved plates and by surface-piercing flat plates. Appl Ocean Res 1994;16:129-39.

[22] Parsons NF, Martin PA. Trapping of water waves by submerged plates using hypersingular integral equations. J Fluid Mech 1995;284:359-75.

[23] Martin PA, Farina L. Radiation of water waves by a heaving submerged horizontal disc. J Fluid Mech 1997;337:365-79.

[24] Farina L, Martin PA. Scattering of water waves by a submerged disc using a hypersingular integral equation. Appl Ocean Res 1998;20:121-34.

[25] Mei CC, Stiassnie M, Yue DK-P. Theory and applications of ocean surface waves. World Scientific; 2005.

[26] Gradshteyn IS, Ryzhik IM. Tables of integrals series and products. Academic Press; 2007

[27] Wei Y, Rafiee A, Henry A, Dias F. Wave interaction with an oscillating wave surge converter, Part I: Viscous Effects; 2014. In revision for Ocean Engineering.

[28] Kolm P, Rokhlin V. Numerical quadratures for singular and hypersingular integrals. Comput Math Appl 2001;41:327-52.

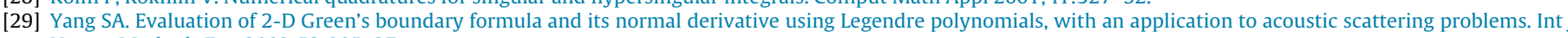
Numer Methods Eng 2002:53:905-27.

[30] Carley M. Numerical quadratures for singular and hypersingular integrals in boundary element methods. Soc Ind Appl Math 2007;29:1207-16.

[31] Kaya AC, Erdogan F. On the solution of integral equations with strongly singular kernels. Q Appl Math 1987;45:105-22. 